\title{
2019 ARIA Care pathways for allergic rhinitis - Egypt
}

Authors: YM El-Gamal, RH El-Owaidy, SS El-Sayed, ZA El-Sayed, EM Hossny, SM Reda, C Bachert, WJ Fokkens, T Haahtela, PH Hellings, L Klimek, N Papadopoulos, N Pham-Thi, O Pfaar, A Valiulis, MT Ventura, G Onorato, W Czarlewski, A Bedbrook, J Bousquet

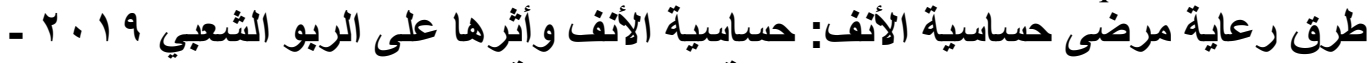 جمهورية مصر العربية الفئية}

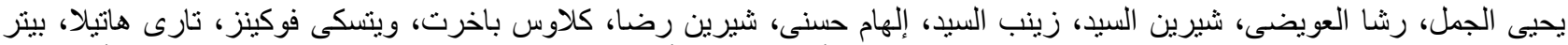

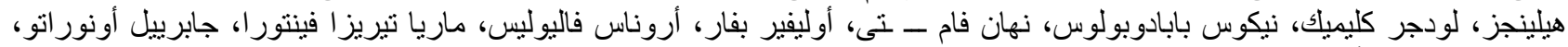

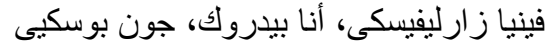
عنوان المر اسلة:

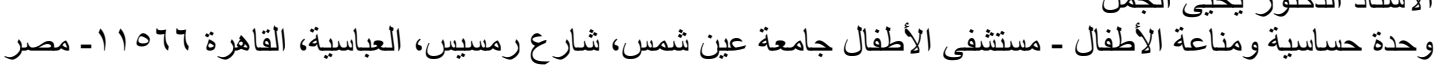

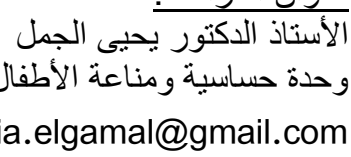

Yehia.elgamal@med.asu.edu.eg; Yehia.elgamal@gmail.com

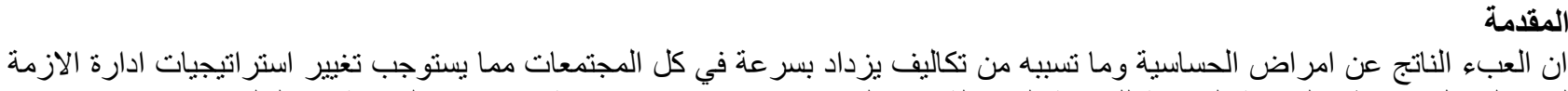

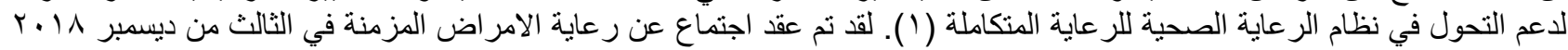

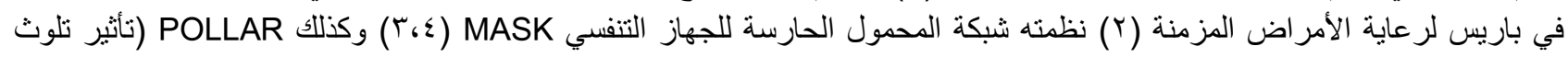

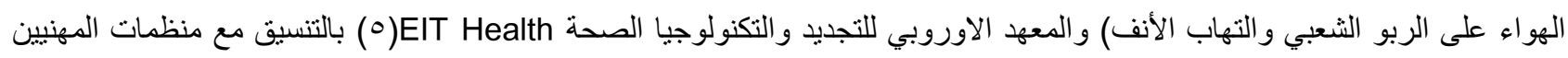

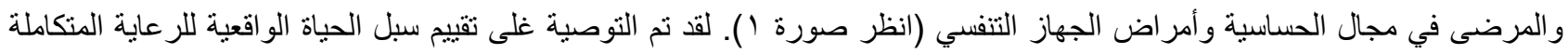
(ICPs)

البيئي(r)

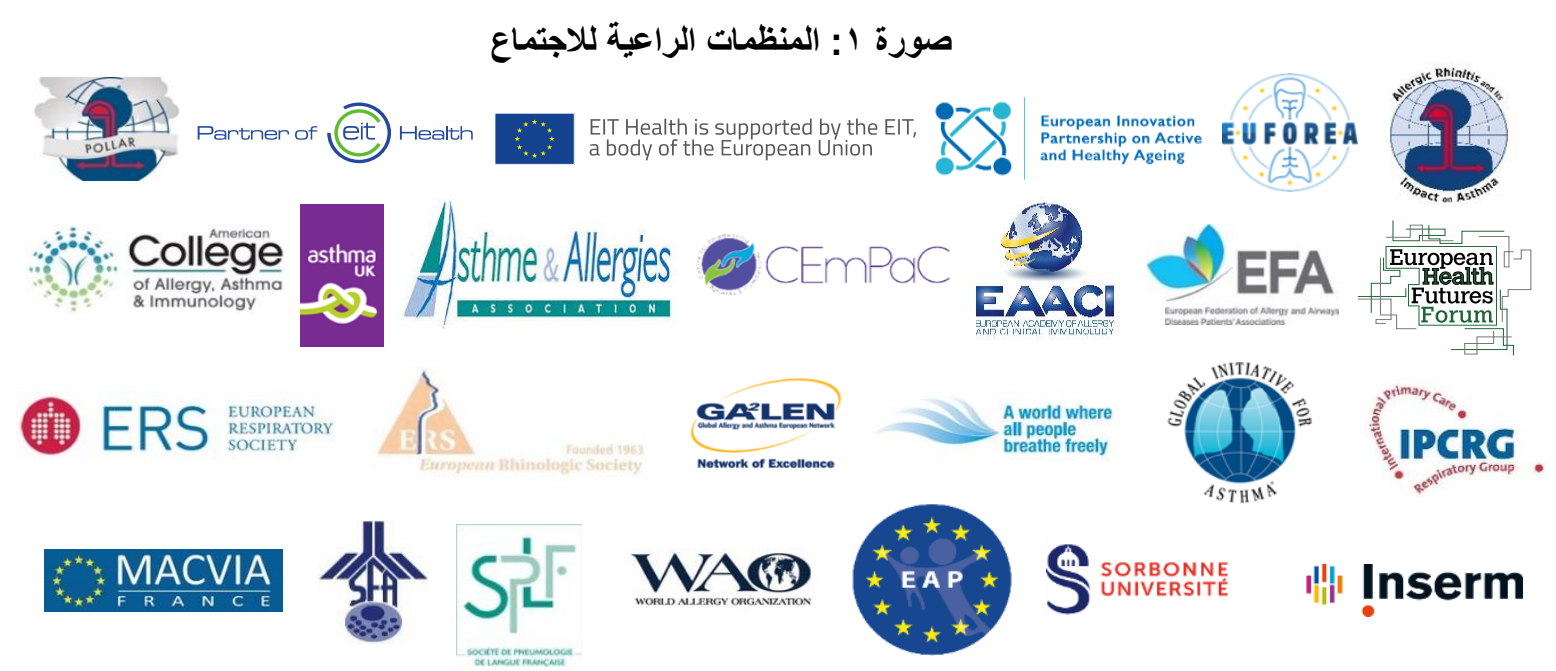

إن سبل الحياة الو اقعية للرعاية المتكاملة (ICPs) هي عبارة عن خطط منظمة متعددة التخصص تعرض تفاصيل الخطوات الرئيسية لرعاية

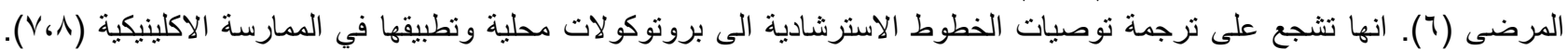

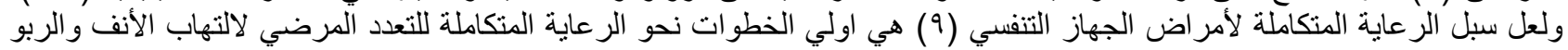

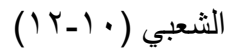
وبالنسبة لحساسية الأنف فهنالك حاجة ملحة لتطوير جيل جديد للعلاج الدو ائي وسبل للرعاية المتكاملة (ICPs) للعلاج المناعي بالاليرجينات لقد نم نشر ورقة عقب اجتماع باريس (rT) (IT). و الاقاليم المختلفة بحيث تكيف التوصيات لتناسب الاستخدام المحلى و المنظومة الصحية. 


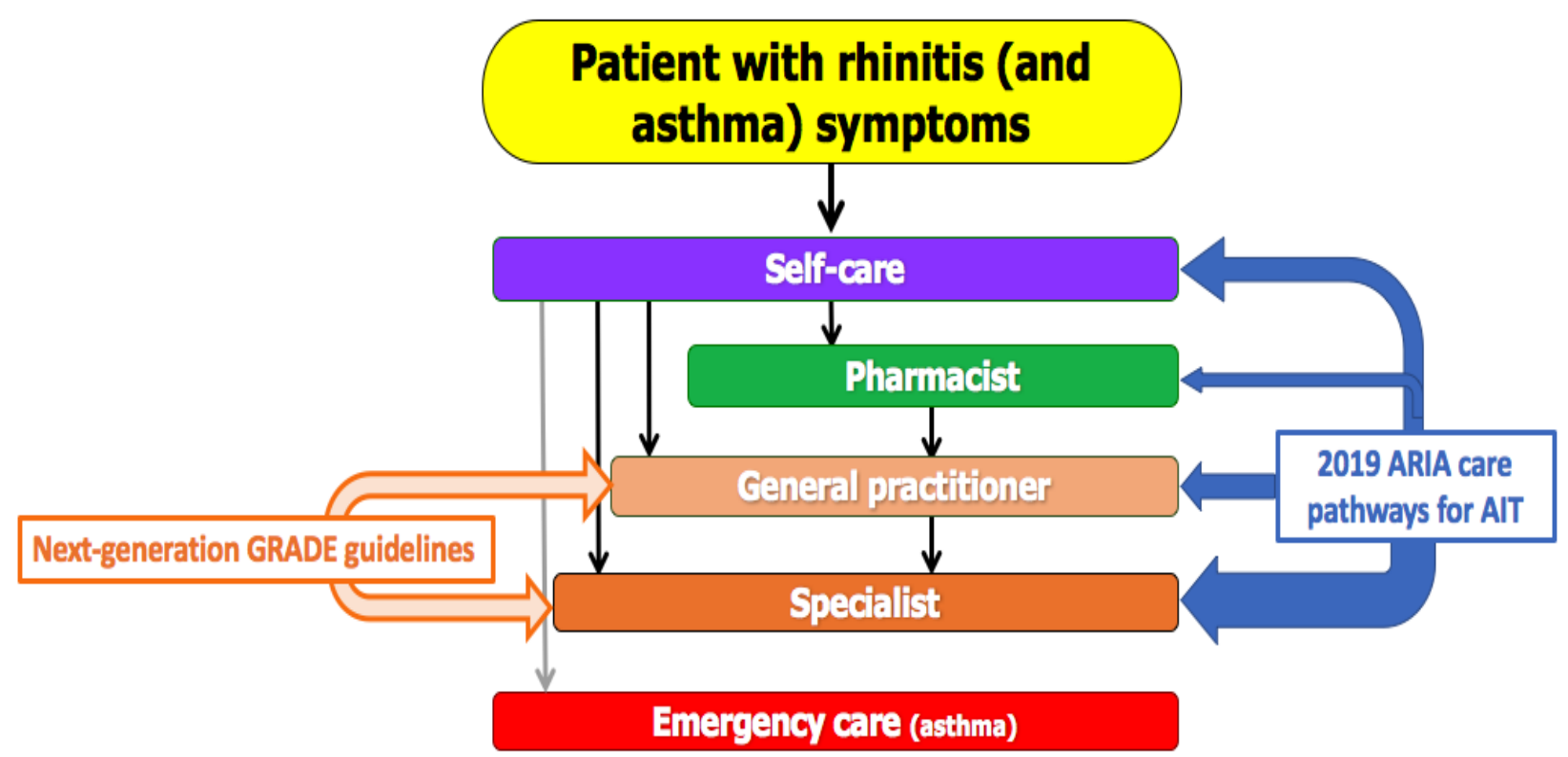

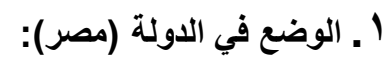

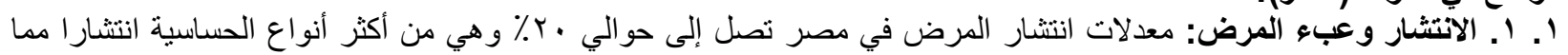

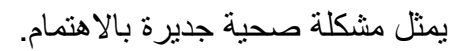

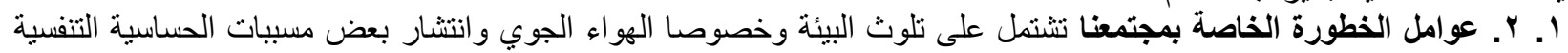

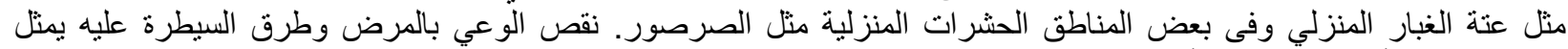

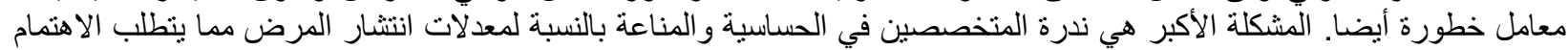

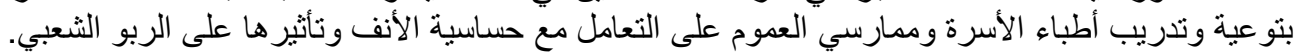

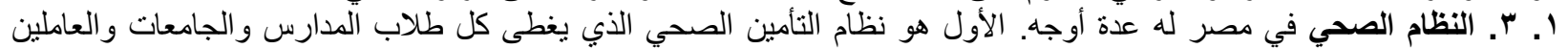

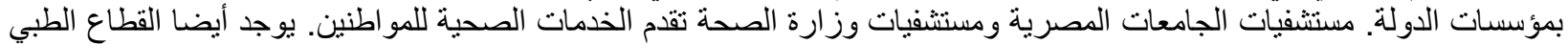

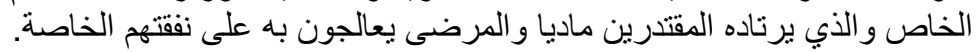

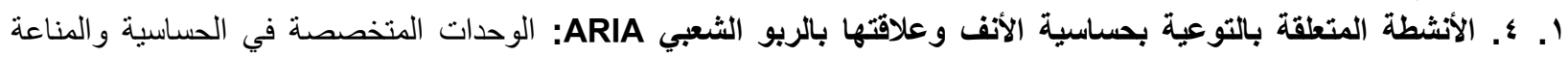

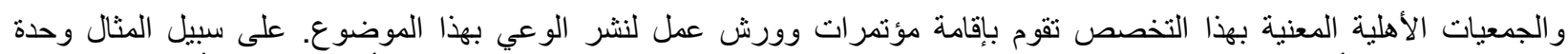

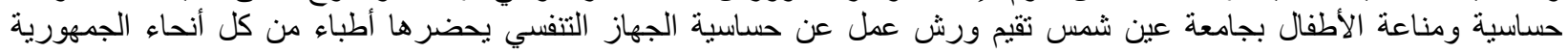

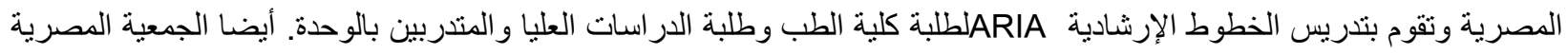

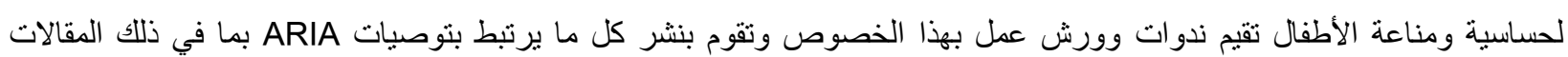

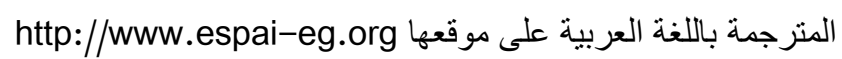

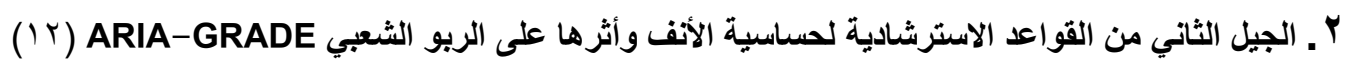

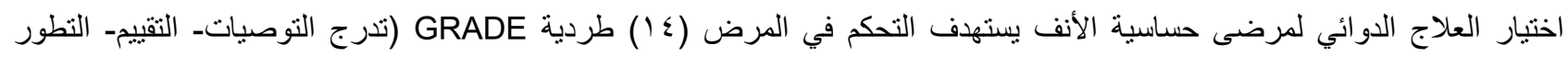

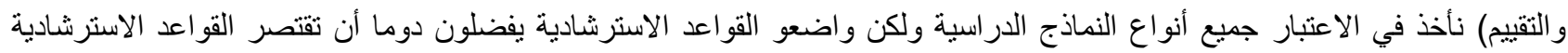

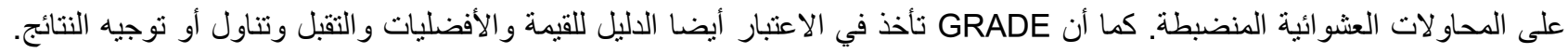

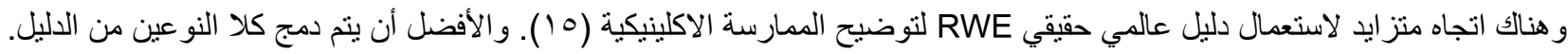

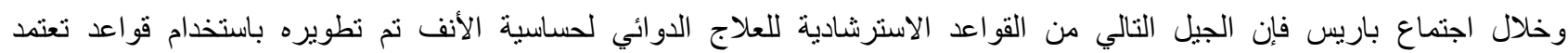

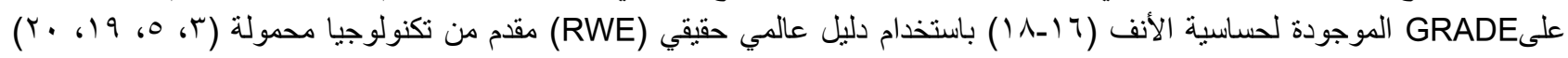

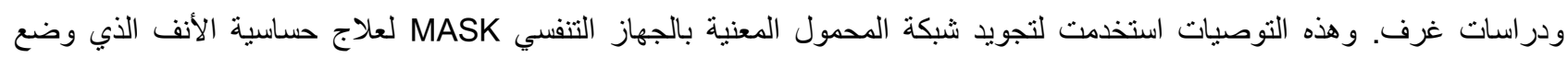

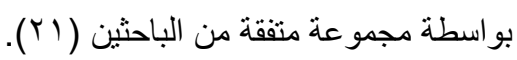




\section{r. 1 ـ الاليل الذي يؤخذ في الاعتبار لتطوير طرق رعاية متكاملة (ICPS) لحساسية الأنف وأثرها على الريو الثعبي r. 1. 1. المنحس MASK للعلاج الدوائي لحساسية الأنف}

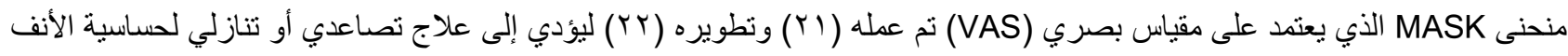

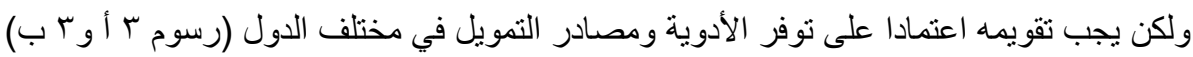

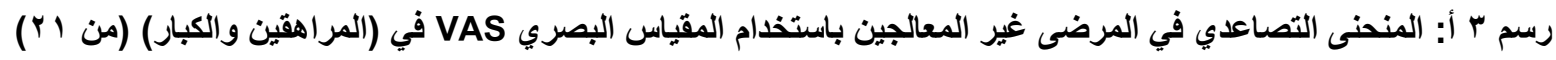

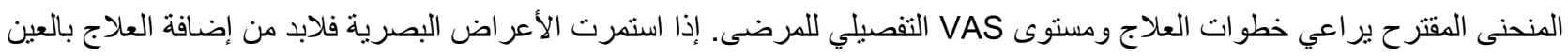
تقييم التحكم في المرضى بأعراض والغير معالجين

\section{Assessment of control in untreated symptomatic patient}

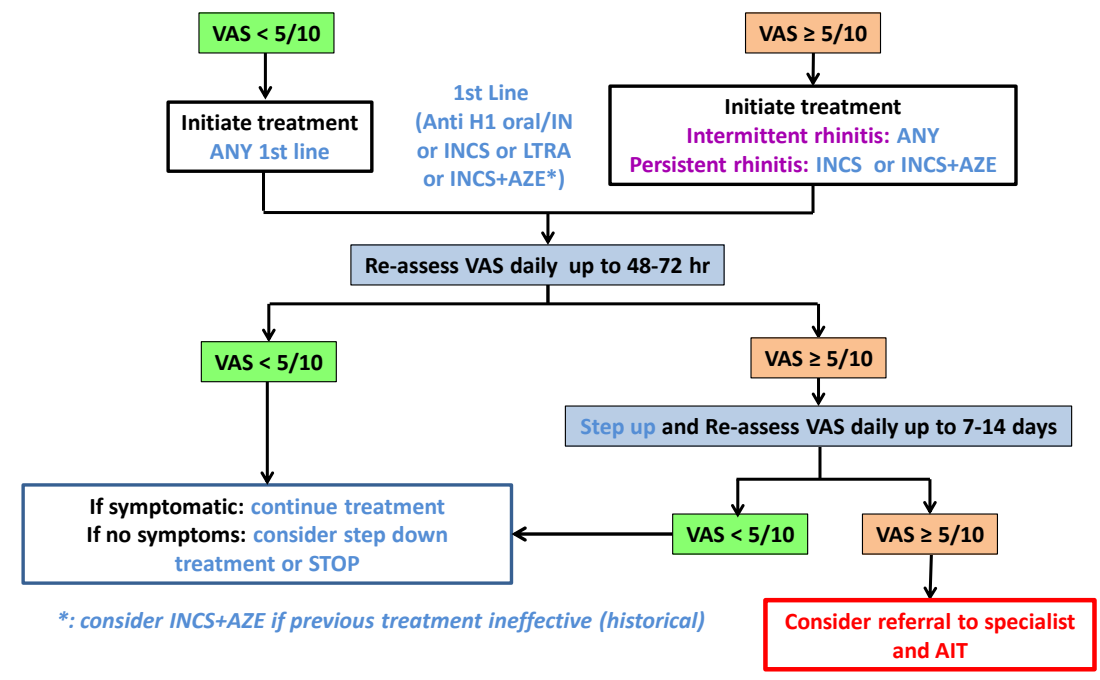

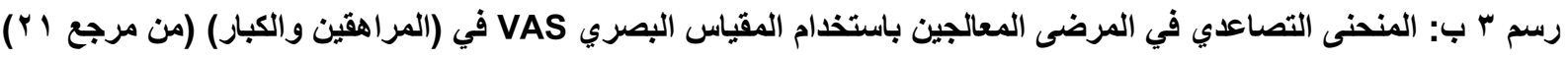

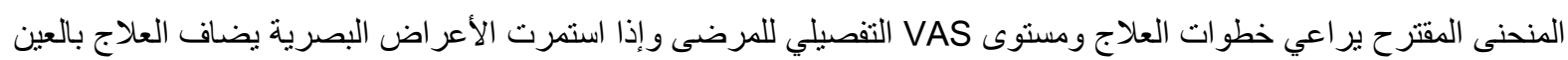
تقييم التحمم في المرض بأعراض ويتلقون العلاج

\section{Assessment of control in treated symptomatic patient}

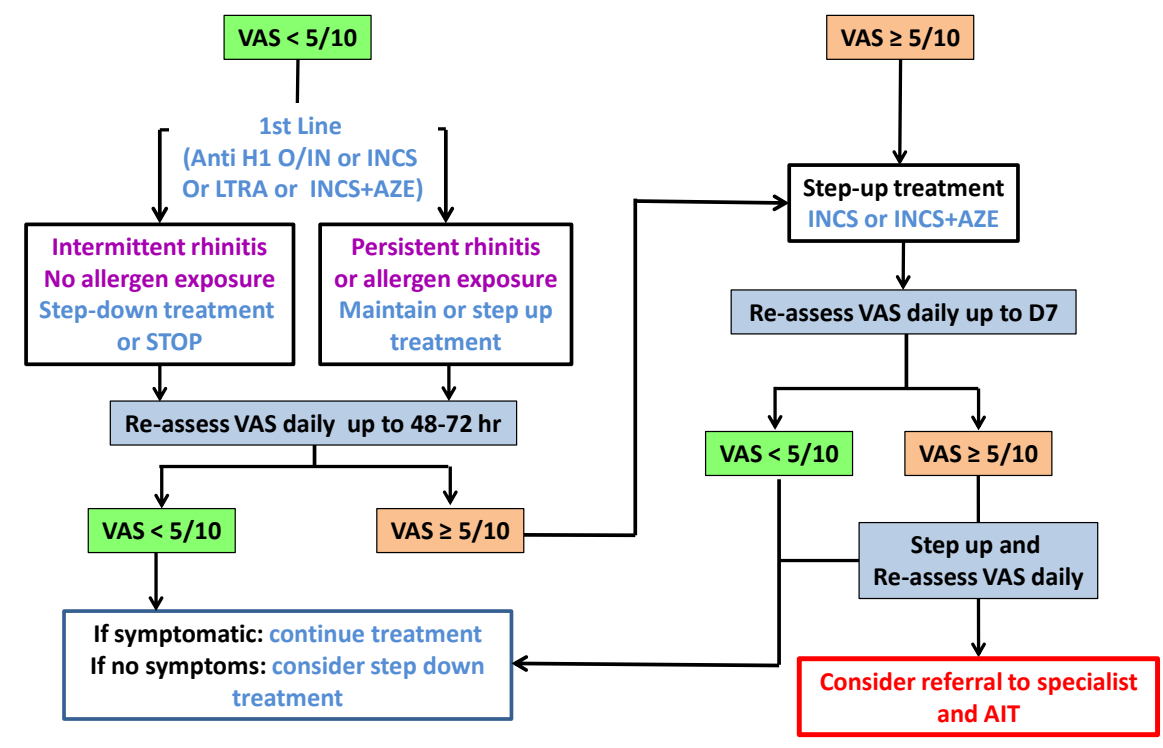




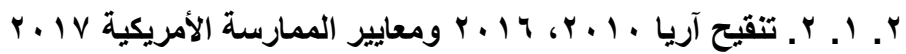

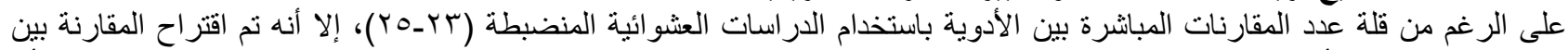

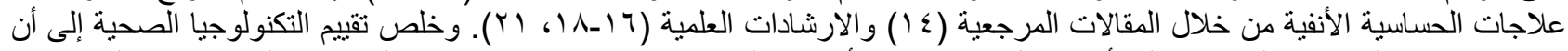

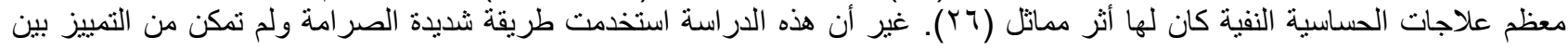
الأدوية .

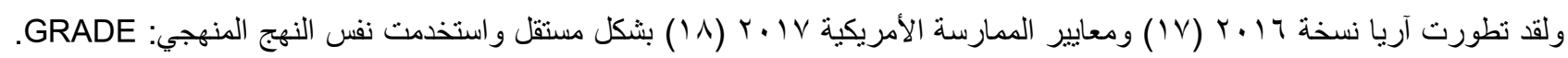

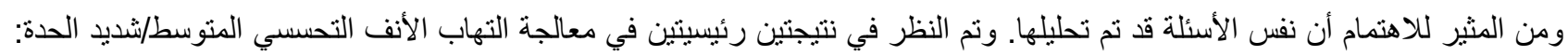

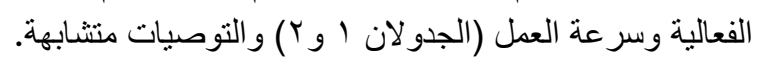

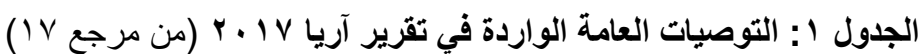

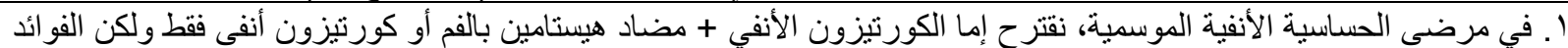

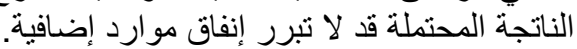

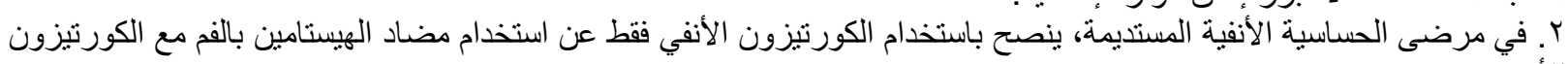

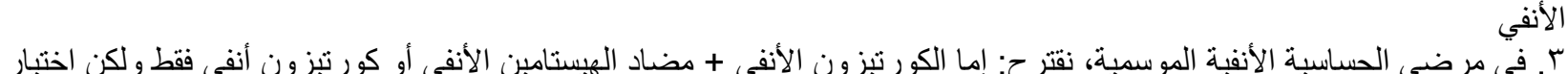

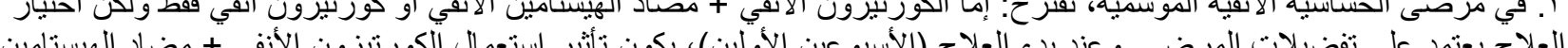

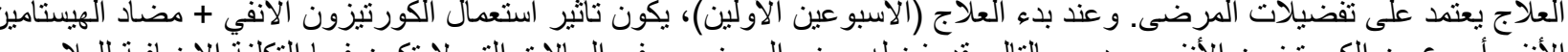

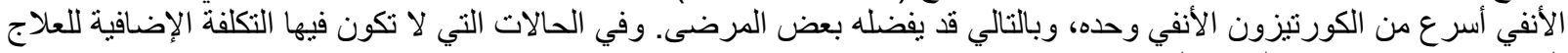

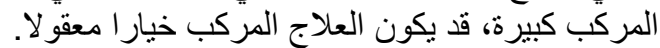

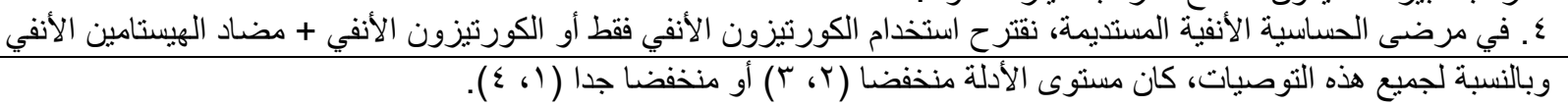

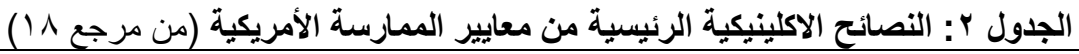

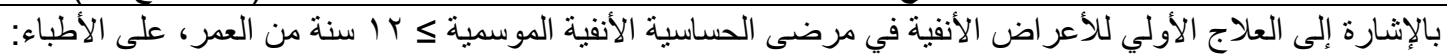

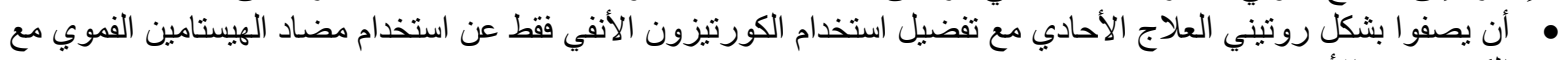

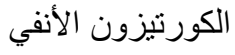

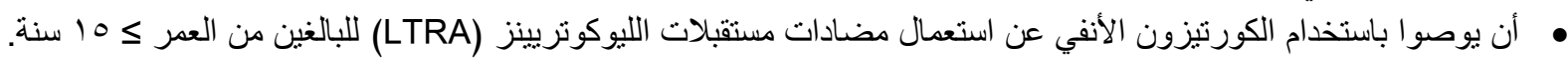

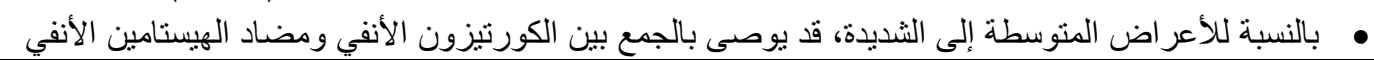

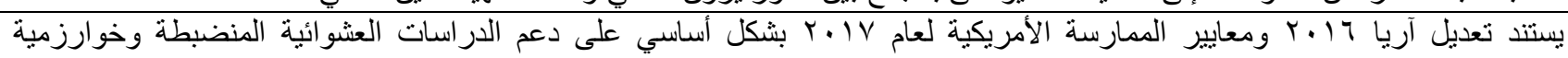

.MASK

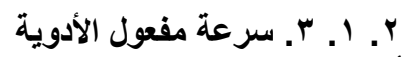

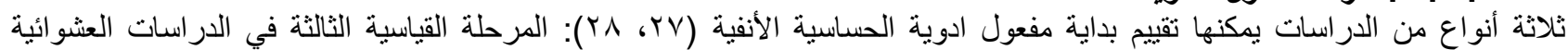

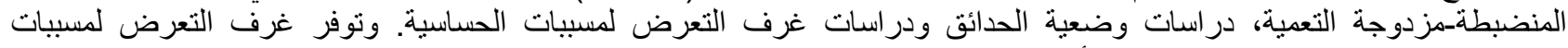

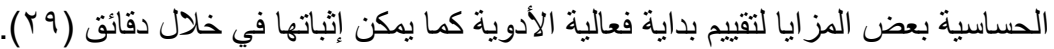

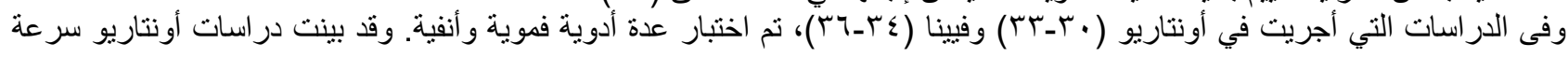

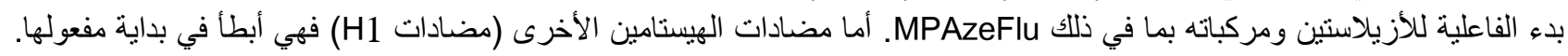

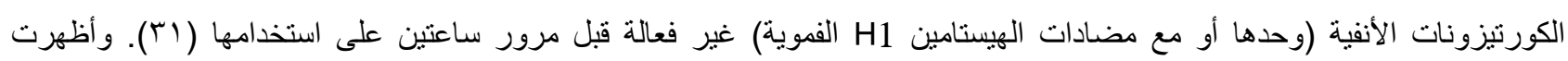

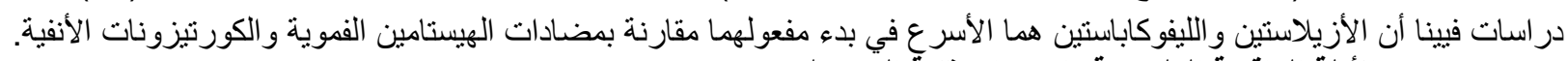

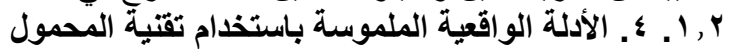

اختبرت الجيل التالي من إرشادات آريا توصيات GRADEباستخدام الأدلة الو اقعية مستخدمة البيانات التي تم الحصول عليها بو اسطة أدوات

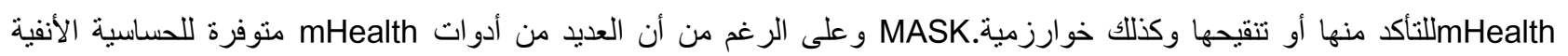

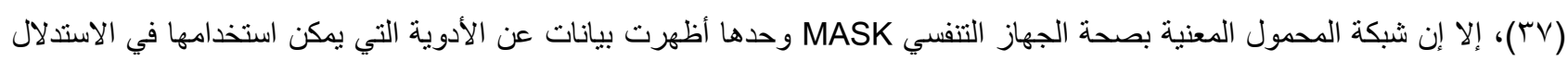

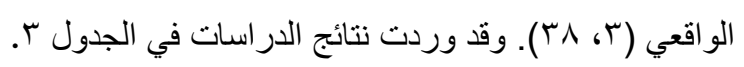

جدول r: نتائج الأدلة الواقعية في علاج الحساسية الأنفية

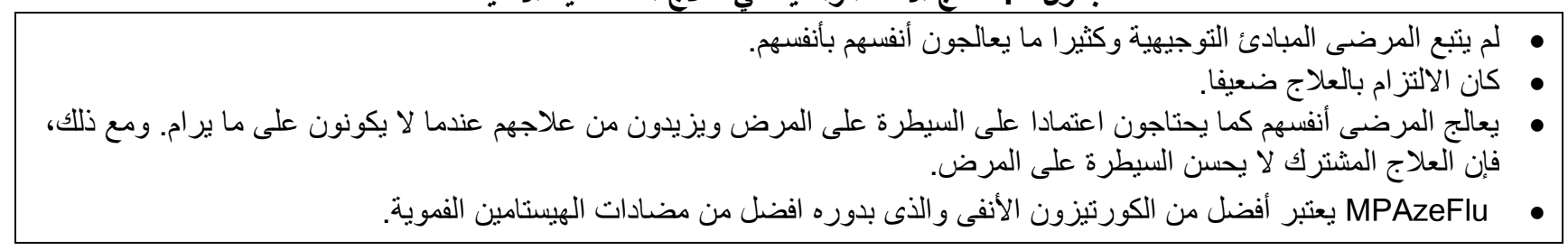


على الرغم من استحالة اثبات الالتزام مباشرة حيث أن مستخدمي MASK لا يفيدون بالبيانات يوميا وقد لا يفيدون أيضا بجميع الأدوية

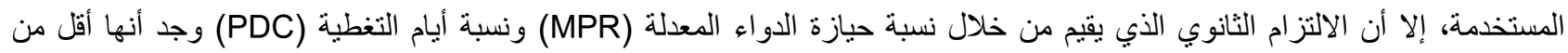

( $(49) 5 \%$

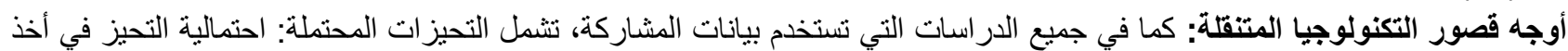

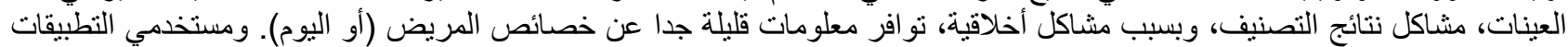
لا يمثلون جميع المرضى المصابين بحساسية الأنف.

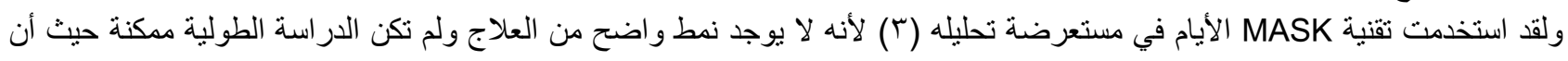

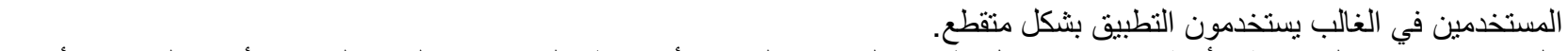
ولم يكن تثخيص الحساسية الأنفية مدعوم من قبل طبيب ولكن من المرجح أن معظم المستخدمين لديهم التهاب الأنف (التحسسي أو غير

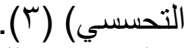
وبالرغم من ذلكى، فإن تكنولو جيا الهو اتف النقالة أصبحت أداة هامة لفهم وإدارة الحساسية الأنفية بشكل أفضل وتضيف معلومات جديدة لم تكن

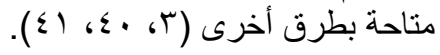
دراسات أخرى عن الأدلة الواقعية باستخدام تكنولوجيا الهاتف النقال: في حد علمنا لا توجد دراسة mHealth أخرى قامت بتقييم فعالية

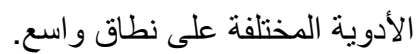

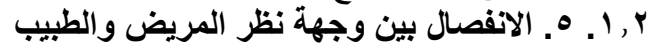

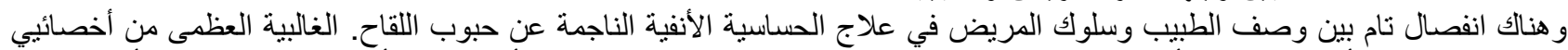

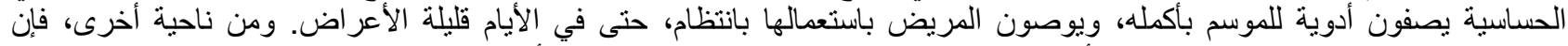

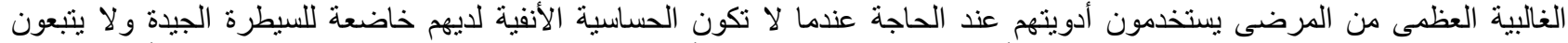

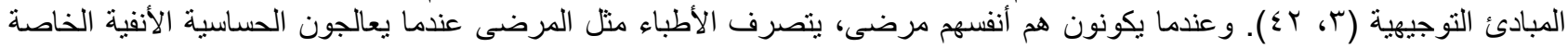

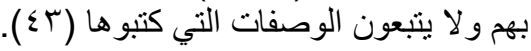

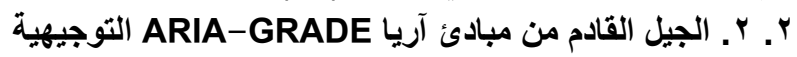
قدمت الخوارزمية المقترحة نهج متدرج لاختيار أدوية الحساسية الأنفية على أساس توصيات المناس المنقدة مع الأدلة الواقعية ودر اسات

جدول ؛ : الجيل القادم من مبادئ آريا التوجيهية

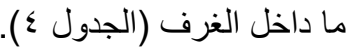

\begin{tabular}{|c|c|c|c|}
\hline | - الغرف & $\begin{aligned} \text { mHealth } \\
\text { الأولدية }\end{aligned}$ & توصيات GRADE & \\
\hline & 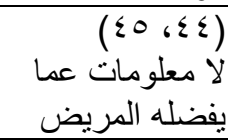 & لا لا معلومات عما يفضله المريض (1) & 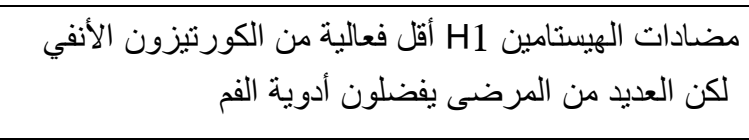 \\
\hline & $(\leqslant 0 ، \leqslant \varepsilon)$ & $(17)$ & الأنفي مضادات الهيستامين الأنفية (H1)أقل فعالية عن الكورنيزون \\
\hline$(\Gamma \leq r \cdot)$ & & $(17)$ & مضادات الهيستامين الأنفية (H1) فعالة في غضون دقائق \\
\hline & $\left(\leqslant 0, r \cdot{ }^{\prime}\right)$ & $(1 \wedge 617)$ & الكورتيزون الأنفي علاج قوى \\
\hline$\left(\sum \tau, r\right)$ & & $(17)$ & 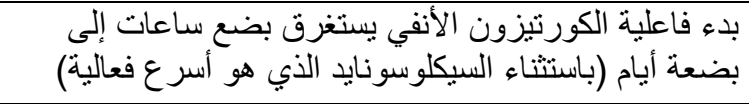 \\
\hline & $(\leqslant 0 ، \leqslant \varepsilon)$ & $(\backslash \wedge, \backslash \vee)$ & 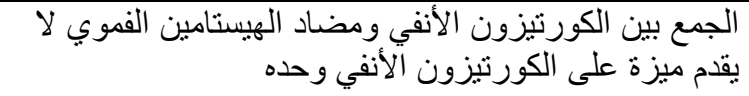 \\
\hline & $(\leqslant 0 ، \leqslant \varepsilon)$ & 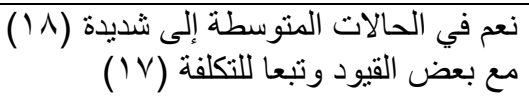 & من الكورتيزون الأنفي وحده الأنفي ومضاد الهيستامين الأنفي أقوى \\
\hline 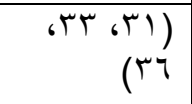 & & & في غضون بين الكورتيزون الأنفي ومضاد الهيستامين الأنفي فعال \\
\hline & & $(1 \wedge, 17)$ & الكورتيزون الأنفيليات اللي وكوتريين أقل فاعلية من \\
\hline
\end{tabular}

ويؤكد النهج المقترح صحة معظم توصيات للحساسية الأنفية، ويسمح بأن تكون الأدلة المشروطة مدعومة بالأدلة الو اقعية كما يقدم

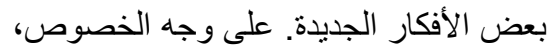
• لم لمتبين أن فعالية مادة مضاد الهيستامين الفموية - H1 مجتمعة مع الكورتيزون الأنفي أكثر فعالية عن الكورتيزون الأنفي وحده. 
•تبين أن كفاءة الجمع بين الكورتيزون الأنفي ومضاد الهيستامين الأنفي تعتبر أقوي من الكورنيزون الأنفي وحده.

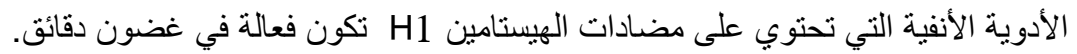

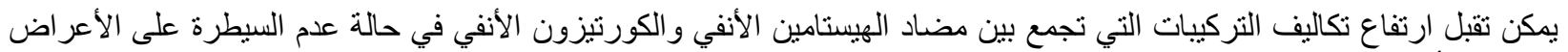

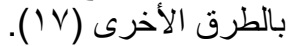

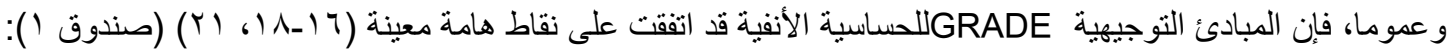
صندوق ا: توصيات للعلاج الدوائي في حالة التهاب الأنف التحسسى:

• مضادات الهيستامين H1 الأنفية أو الفموية تعتبر أقل فعالية عن الكورتيزون الأنفي للسيطرة على جميع أعر اض التهاب الحساسية الأنفية

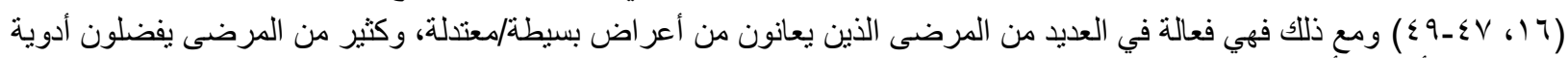
بالفم على الأدوية الأنفية

ه تختلف المقارنات بين مضادات الهيستامين H1 الفموية والأنفية بين التوصيات ولم يتم التوصل إلى استتتاجات محددة. • في المرضى الذين يعانون من الحساسية الأنفية الثديدة، يمثل الكورتيزون الأنفي علاج الخط الأول. غير أنه يحتاج إلى بضعة أيام لكي يكون فعال تماما.

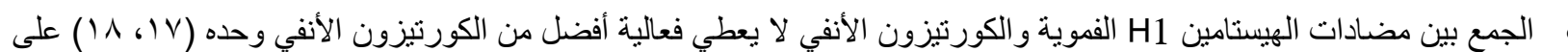
الرغم من أن هذه الممارسة شائعة عالميا.

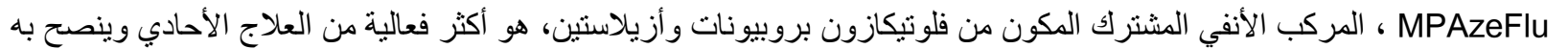

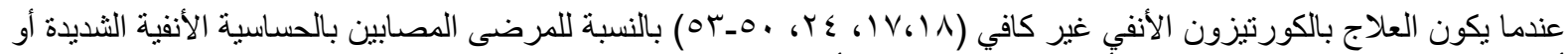

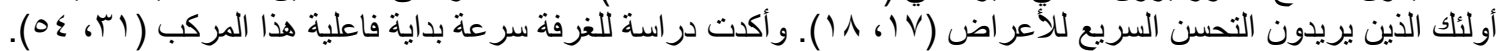
تعتبر جميع الأدوية الموصي بها آمنة في الجرعة المعتادة. الجيل الأول من مضادات الهيستامين H1الفموية لها لأتئير مهدئ ومنوم وينبغي تجنبها (00) بالإضافة إلى تجنب الاستخدام المطول لقابض الأبه الأوعية الأنفية.

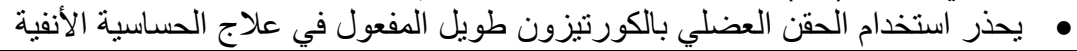
الاستتتاجات

تم اختبار خوارزمبة آريا من خلال در اسات عشو ائية منضبطة (RCTs)، ودر اسات غرف الحساسية وبحوث الأدلة الو اقعية. وقد تبين أن

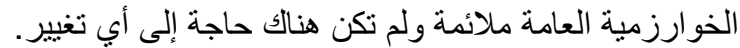

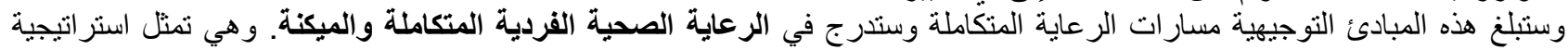
إدارة التغيير للمرحلة الرابعة من آريا ( ). (1).

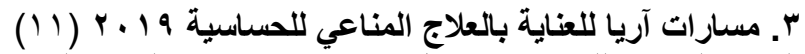

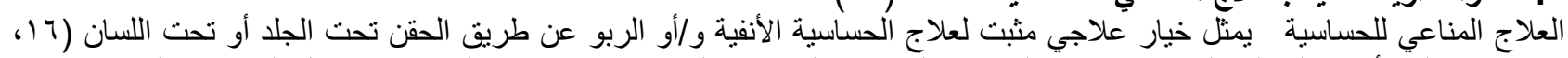

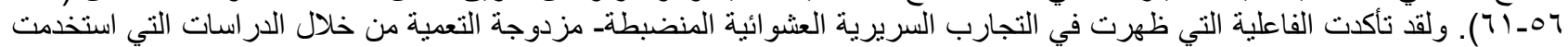

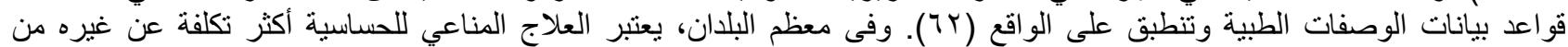

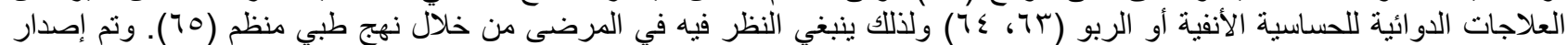

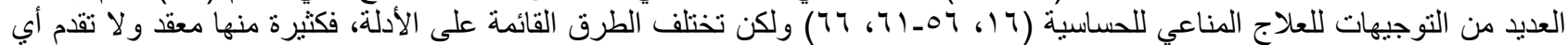

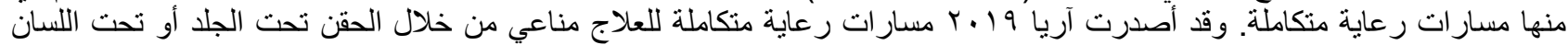
(1) ملخصه هنا في هذه المقالة.

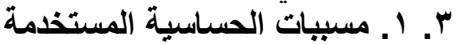
• المستخلص ذأو الصلة: ينبغي أن يستتد قر ار وصف العنات العلاج المناعي للحساسية إلى الأعر اض أثناء التعرض للحساسية، و إثبات التحسس،

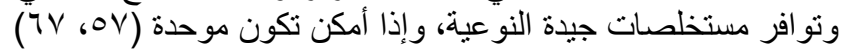

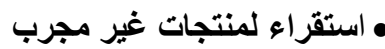

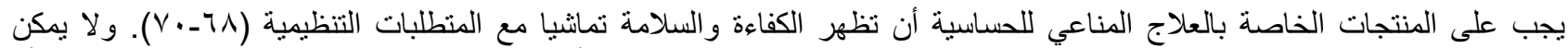

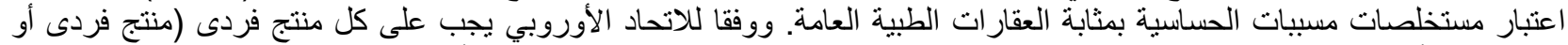

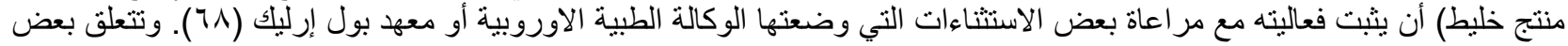

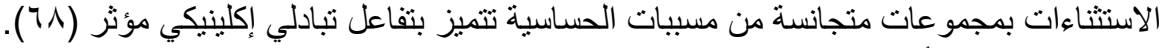

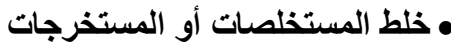

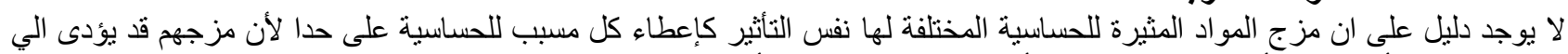

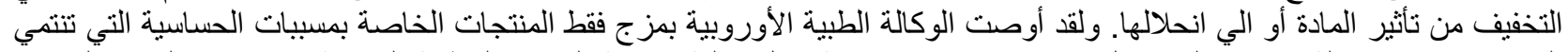

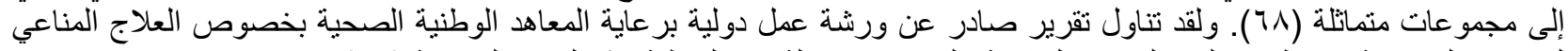

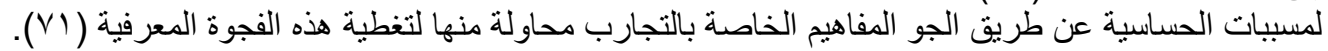


• منتجات للمريض مسماه باسمه لـات

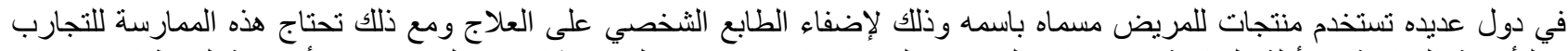
التأكيدية المناسبة والأدلة الوافية اقعية. ومنتجات المريض المسماة باسمه تسوق على استثناء من التشريعات الأوروبية لمقتطفات مسببات

يعاني مرضى الحساسية من التحسس من كثير من التئ المواد المثيرة للحساسية عن طريق الجلوبيولين المناعي ولكن ليس كل تحسس من مادة

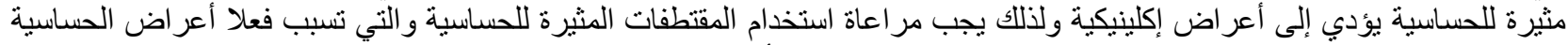

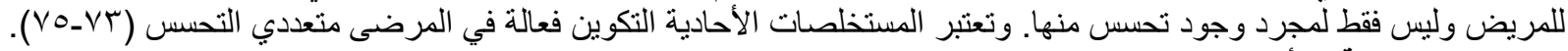

r. r. r. r. السلامة والأمان:

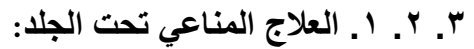

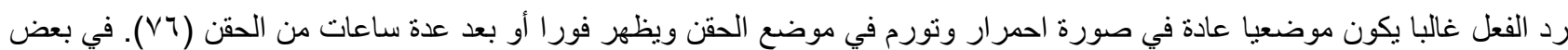

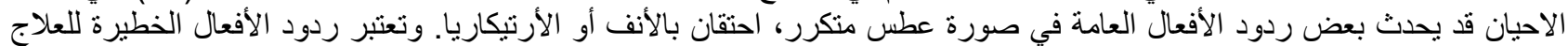

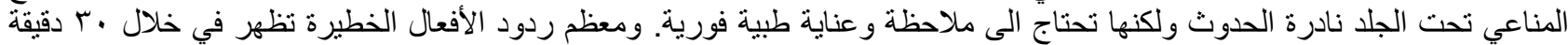

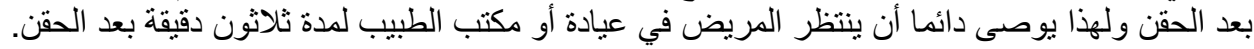

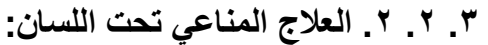

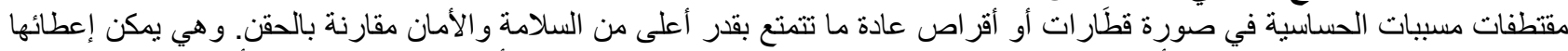

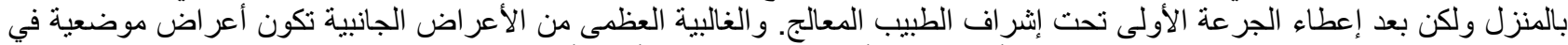

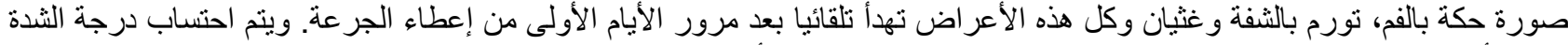

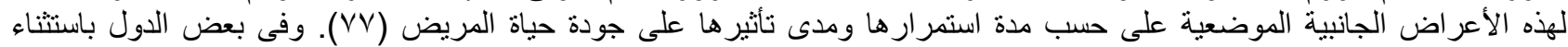

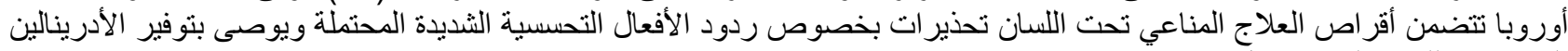

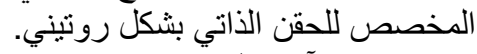

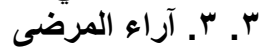

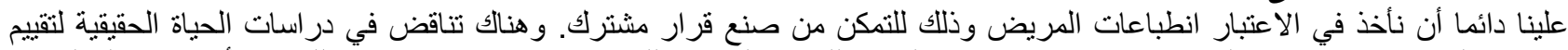

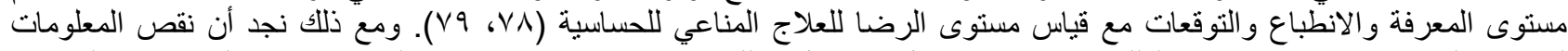

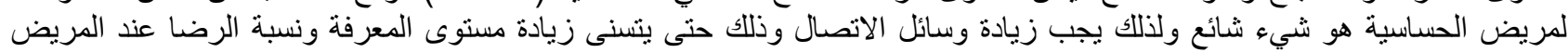

( $(\Lambda)$ ، $\wedge \cdot)$

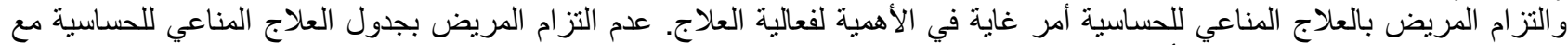

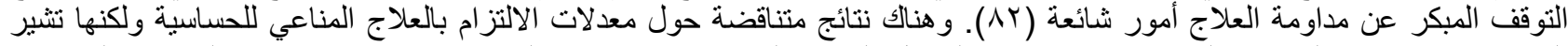

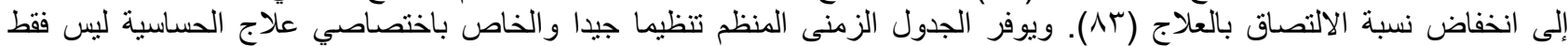

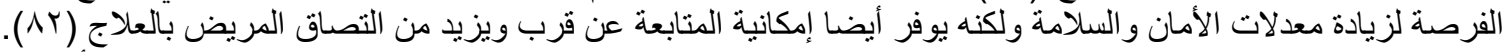

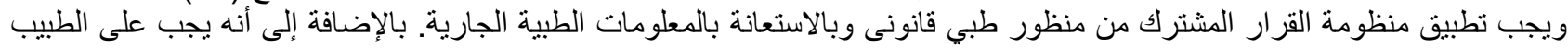

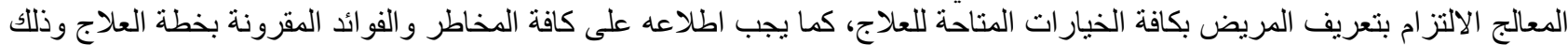

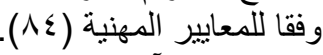

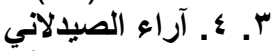

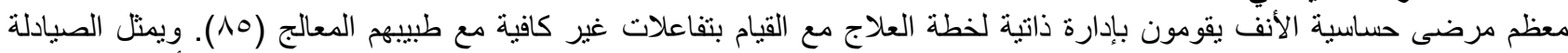

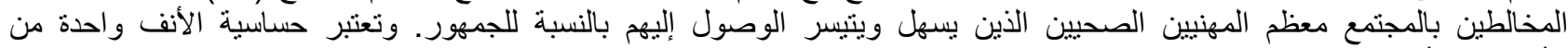

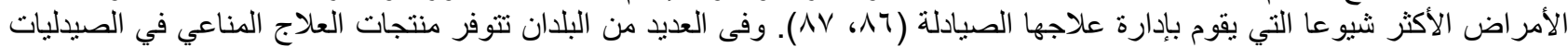

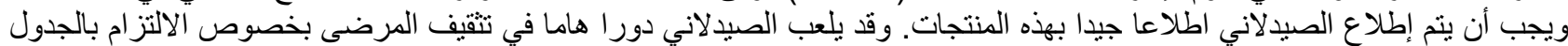

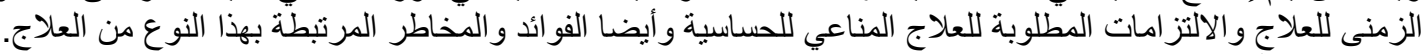

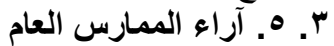

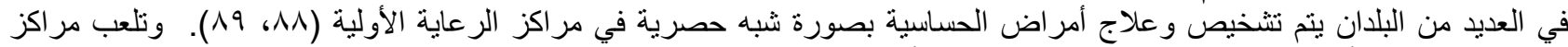

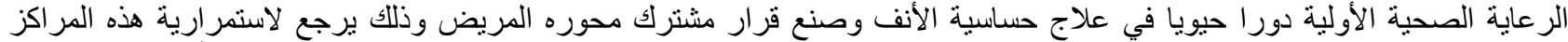

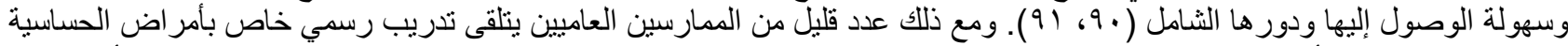

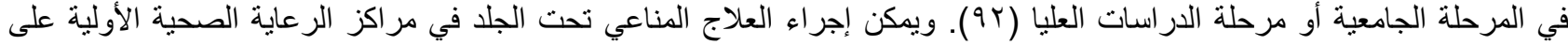

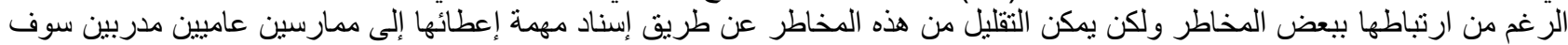

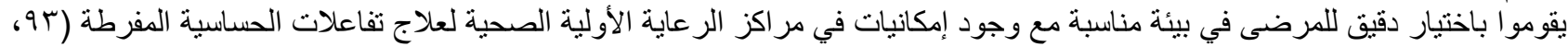

r.

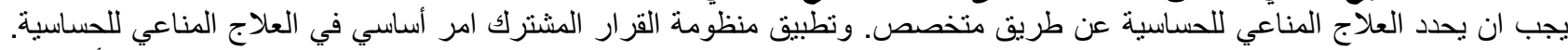

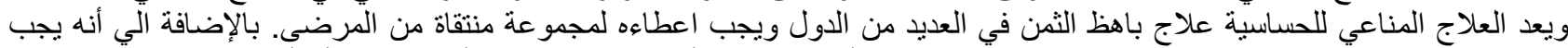

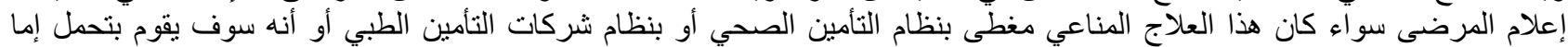
جز عن التكاليف او تحمل تكاليف العلاج كاملَّة. 


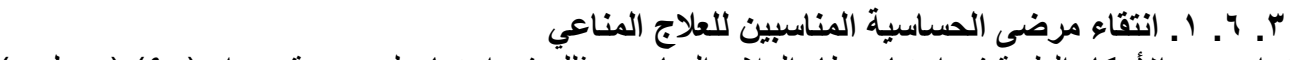

لقد تم اقتر اح دور الأحكام الطبية في اختبار نظام العلاج المناعي وذلكاع في اجتماع لمجموعة خبراء (90) (جدول 0).

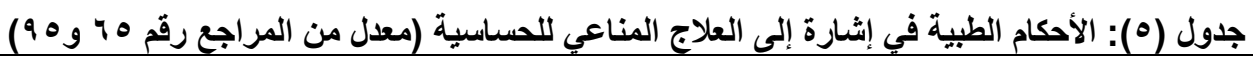

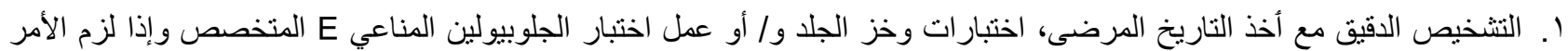

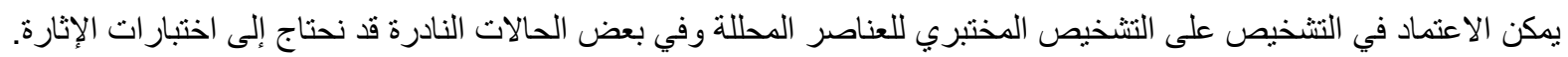

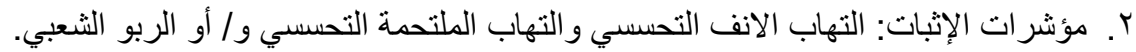

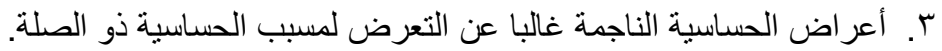

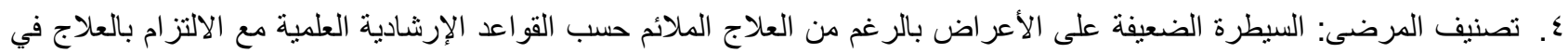

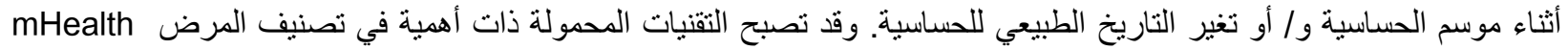

biomarker

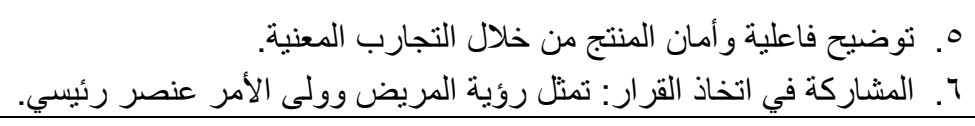

ولقد تم اقتراح اتخاذ نهج الطب الدقيق في أمراض الحساسية (الرسم ع) (70، 90). فقي بعض الأحوال، يمكن تقديم العلاج المناعي

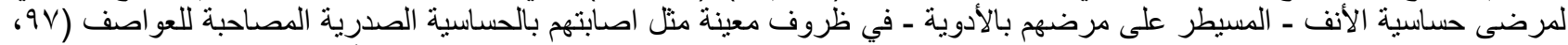

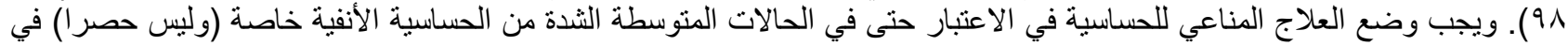

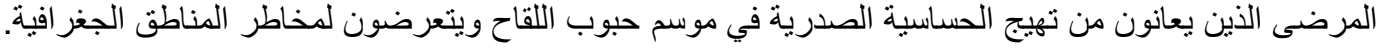

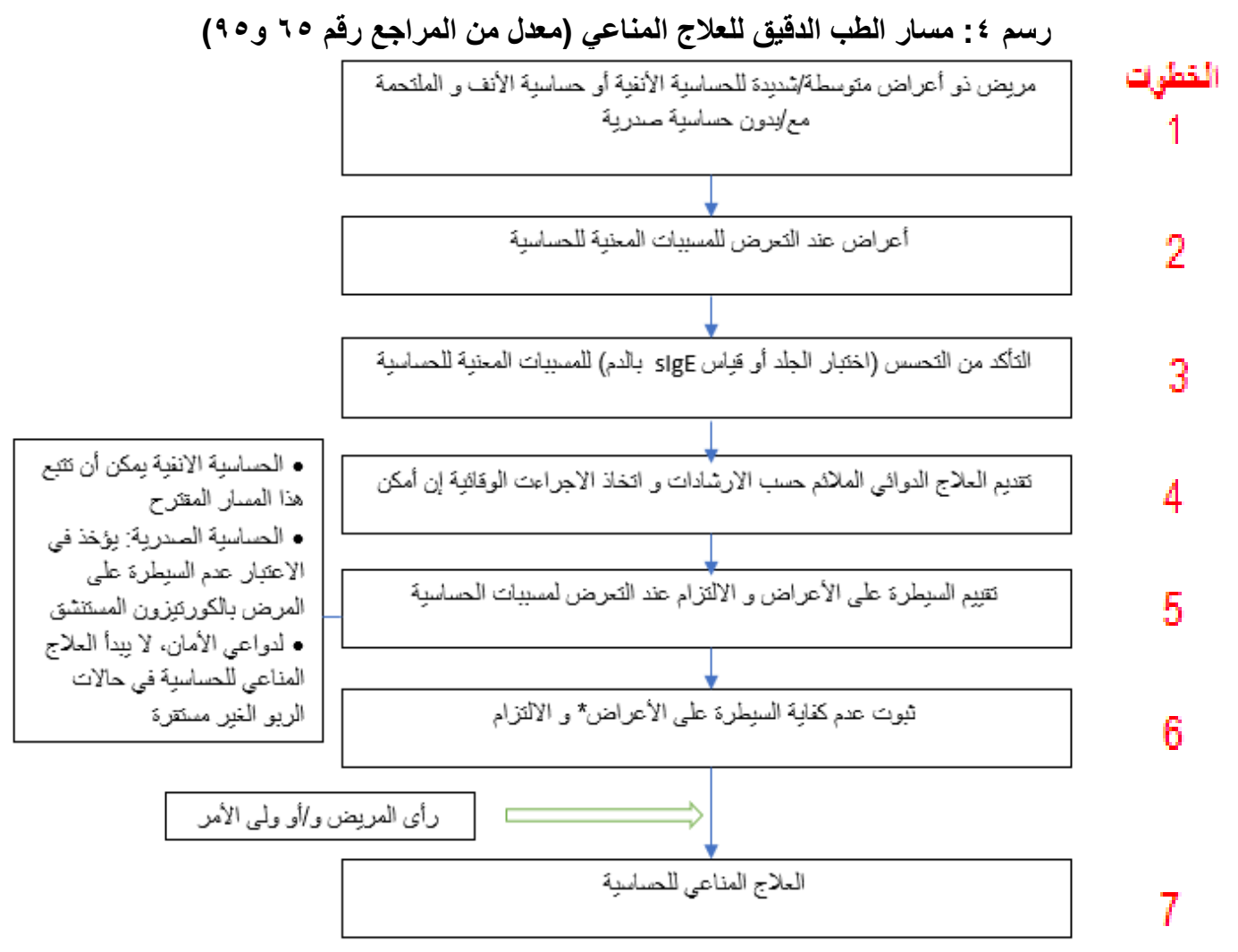

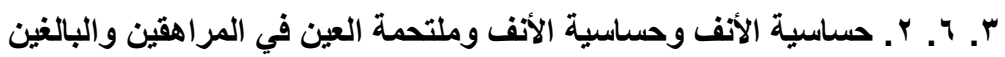

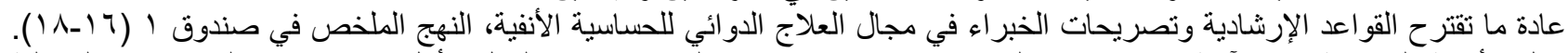

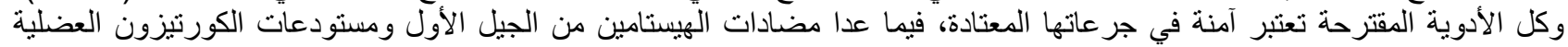

و التي يجب الادبة النبها (00) نئر

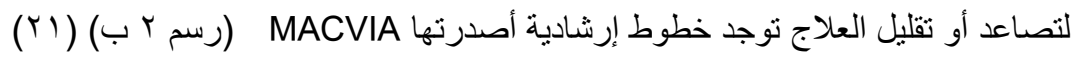




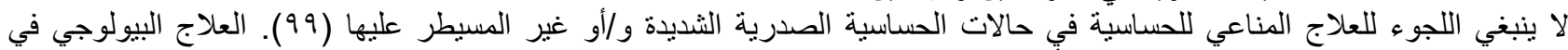

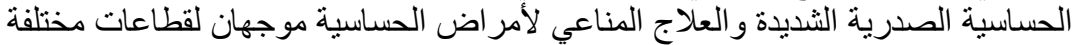

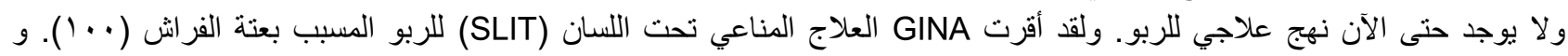

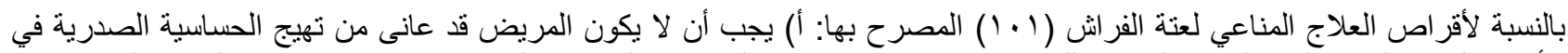

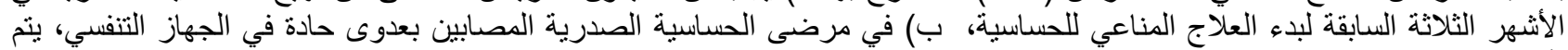

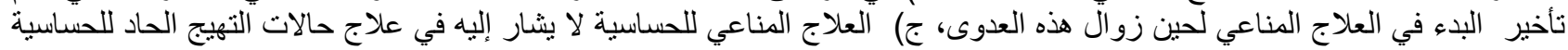

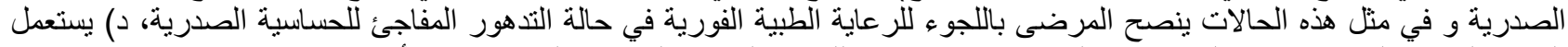

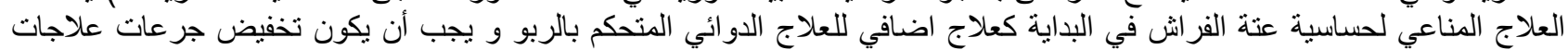

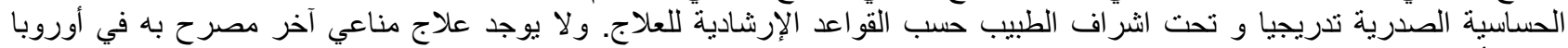
كعلاج أولي للحساسية الصدرية.

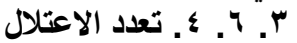

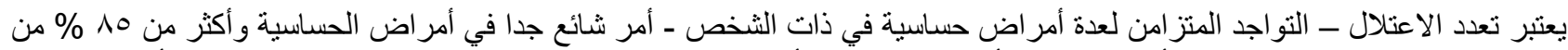

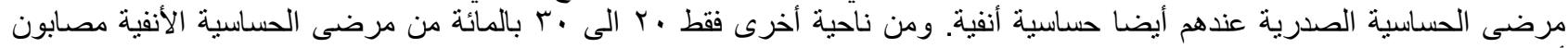

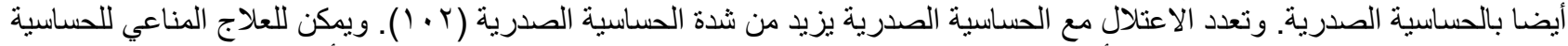

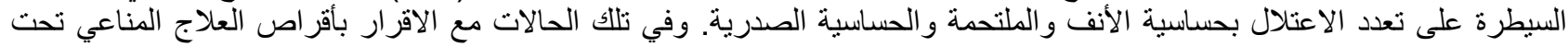

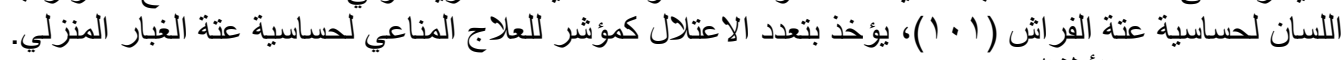

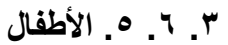

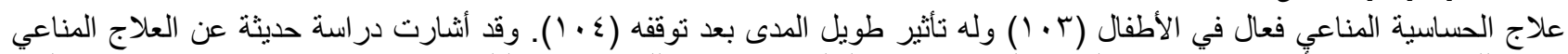

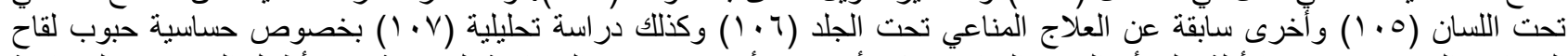

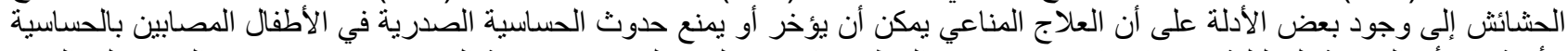

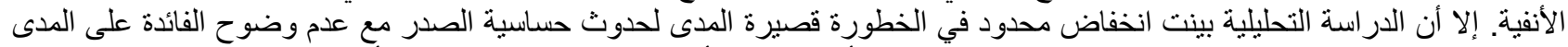

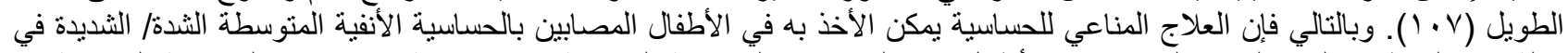

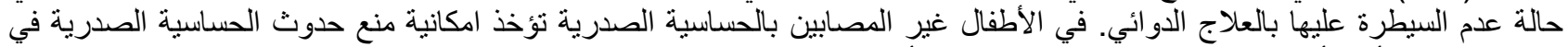

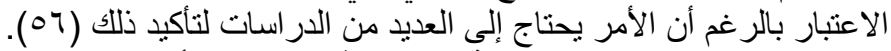
r. 7 . 7.

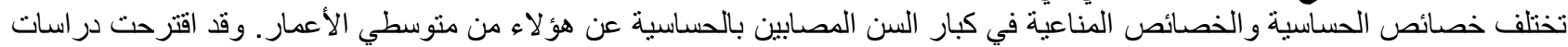

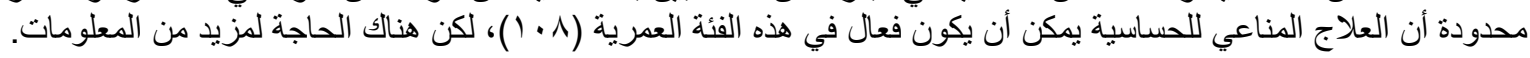

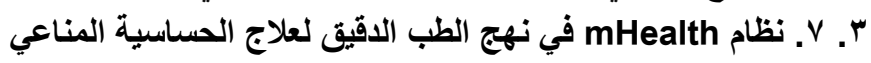

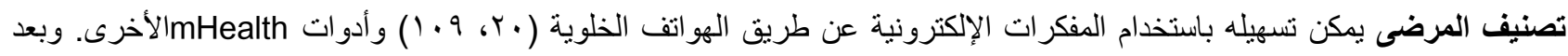

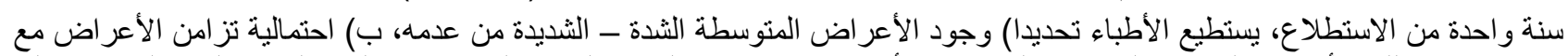

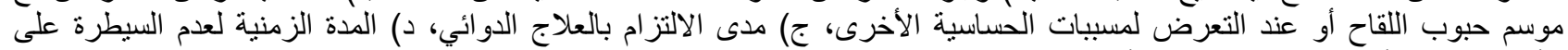

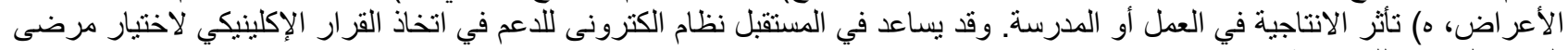

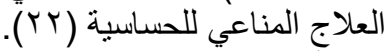
متابعة المرضى الخاضعين للعلاج المناعي للحساسية: يمكن اتخاذ نفس النهج لمتابعة المرضى الخاضعين للعلاج المناعي للحساسية لتقيبم مدة فاعليته (·) (1). الخلاصة: الماعلة

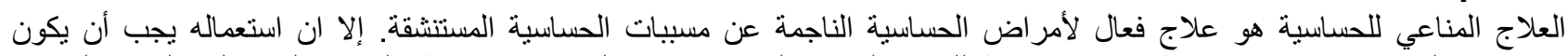
مقصور العلى حالات مختارة بعناية و غير مستجيبة للعلاج الدوائي الملائم وحسب القئ القوات اعد الإرشادية ولهؤلاء المتاح لهم العلاج المناعي الفعال مقارنة بالتكلفة.

\section{REFERENCES}

1. Bousquet J, Hellings PW, Agache I, Amat F, Annesi-Maesano I, Ansotegui IJ, et al. Allergic Rhinitis and its Impact on Asthma (ARIA) Phase 4 (2018): Change management in allergic rhinitis and asthma multimorbidity using mobile technology. J Allergy Clin Immunol. 2019;143(3):864-79.

2. Bousquet JJ, Schunemann HJ, Togias A, Erhola M, Hellings PW, Zuberbier T, et al. Next-generation ARIA care pathways for rhinitis and asthma: a model for multimorbid chronic diseases. Clin Transl Allergy. 2019;9:44.
3. Bousquet J, Arnavielhe S, Bedbrook A, Bewick M, Laune D, Mathieu-Dupas E, et al. MASK 2017: ARIA digitally-enabled, integrated, person-centred care for rhinitis and asthma multimorbidity using real-world-evidence. Clin Transl Allergy. 2018;8:45.

4. Bousquet J, Bedbrook A, Czarlewski W, Onorato GL, Arnavielhe S, Laune D, et al. Guidance to 2018 good practice: ARIA digitally-enabled, integrated, person-centred care for rhinitis and asthma. Clin Transl Allergy. 2019;9:16. 
5. Bousquet J, Anto JM, Annesi-Maesano I, Dedeu T, Dupas E, Pepin JL, et al. POLLAR: Impact of air POLLution on Asthma and Rhinitis; a European Institute of Innovation and Technology Health (EIT Health) project. Clin Transl Allergy. 2018;8:36.

6. Campbell H, Hotchkiss R, Bradshaw N, Porteous M. Integrated care pathways. BMJ. 1998;316(7125):133-7.

7. Hujala A, Taskinen H, Rissanen S. In: Richardson E, van Ginneken E, editors. How to support integration to promote care for people with multimorbidity in Europe? European Observatory Policy Briefs. Copenhagen (Denmark)2017.

8. Palmer K, Marengoni A, Forjaz MJ, Jureviciene E, Laatikainen T, Mammarella F, et al. Multimorbidity care model: Recommendations from the consensus meeting of the Joint Action on Chronic Diseases and Promoting Healthy Ageing across the Life Cycle (JA-CHRODIS). Health Policy. 2018;122(1):4-11.

9. Bousquet J, Addis A, Adcock I, Agache I, Agusti A, Alonso A, et al. Integrated care pathways for airway diseases (AIRWAYS-ICPs). Eur Respir J. 2014;44(2):304-23.

10. Bosnic-Anticevich S, Costa E, Menditto E, Lourenco O, Novellino E, Bialek S, et al. ARIA pharmacy 2018 "Allergic rhinitis care pathways for community pharmacy". Allergy. 2019.

11. Bousquet J, Pfaar O, Togias A, Schunemann HJ, Ansotegui I, Papadopoulos NG, et al. 2019 ARIA Care pathways for allergen immunotherapy. Allergy. 2019.

12. Bousquet J, Schünemann H, Togias A, Bachert C, Erhola M, Hellings P, et al. Next-generation ARIA guidelines for allergic rhinitis based on GRADE and real-world evidence. J Allergy Clin Immunol. 2019; in press.

13. Bousquet J, Pham-Thi N, Bedbrook A, Agache I, Annesi-Maesano I, Ansotegui I, et al. Nextgeneration care pathways for allergic rhinitis and asthma multimorbidity: a model for multimorbid non-communicable diseases-Meeting Report (Part 1). J Thorac Dis. 2019;11(8):3633-42.

14. Meltzer EO, Wallace D, Dykewicz M, Shneyer L. Minimal Clinically Important Difference (MCID) in Allergic Rhinitis: Agency for Healthcare Research and Quality or Anchor-Based Thresholds? J Allergy Clin Immunol Pract. 2016;4(4):682-8 e6.

15. Sherman RE, Anderson SA, Dal Pan GJ, Gray GW, Gross T, Hunter NL, et al. Real-World Evidence What Is It and What Can It Tell Us? N Engl J Med. 2016;375(23):2293-7.

16. Brozek JL, Bousquet J, Baena-Cagnani CE, Bonini $\mathrm{S}$, Canonica GW, Casale TB, et al. Allergic Rhinitis and its Impact on Asthma (ARIA) guidelines: 2010 revision. J Allergy Clin Immunol. 2010;126(3):46676.
17. Brozek JL, Bousquet J, Agache I, Agarwal A, Bachert C, Bosnic-Anticevich S, et al. Allergic Rhinitis and its Impact on Asthma (ARIA) Guidelines - 2016 Revision. J Allergy Clin Immunol. 2017;140(4):950-8.

18. Dykewicz MS, Wallace DV, Baroody F, Bernstein J, Craig T, Finegold I, et al. Treatment of seasonal allergic rhinitis: An evidence-based focused 2017 guideline update. Ann Allergy Asthma Immunol. 2017;119(6):489-511 e41.

19. Bousquet J, Devillier P, Anto JM, Bewick M, Haahtela T, Arnavielhe S, et al. Daily allergic multimorbidity in rhinitis using mobile technology: A novel concept of the MASK study. Allergy. 2018;73(8):1622-31.

20. Bousquet J, Devillier P, Arnavielhe S, Bedbrook A, Alexis-Alexandre G, van Eerd M, et al. Treatment of allergic rhinitis using mobile technology with realworld data: The MASK observational pilot study. Allergy. 2018;73(9):1763-74.

21. Bousquet J, Schunemann HJ, Hellings PW, Arnavielhe S, Bachert C, Bedbrook A, et al. MACVIA clinical decision algorithm in adolescents and adults with allergic rhinitis. J Allergy Clin Immunol. 2016;138(2):367-74 e2.

22. Courbis AL, Murray RB, Arnavielhe S, Caimmi D, Bedbrook A, Van Eerd M, et al. Electronic Clinical Decision Support System for allergic rhinitis management: MASK e-CDSS. Clin Exp Allergy. 2018;48(12):1640-53.

23. Horak F, Bruttmann G, Pedrali P, Weeke B, Frolund L, Wolff $\mathrm{HH}$, et al. A multicentric study of loratadine, terfenadine and placebo in patients with seasonal allergic rhinitis. Arzneimittelforschung. 1988;38(1):124-8.

24. Hampel FC, Ratner PH, Van Bavel J, Amar NJ, Daftary P, Wheeler W, et al. Double-blind, placebocontrolled study of azelastine and fluticasone in a single nasal spray delivery device. Ann Allergy Asthma Immunol. 2010;105(2):168-73.

25. Kaszuba SM, Baroody FM, deTineo M, Haney L, Blair C, Naclerio RM. Superiority of an intranasal corticosteroid compared with an oral antihistamine in the as-needed treatment of seasonal allergic rhinitis. Arch Intern Med. 2001;161(21):2581-7.

26. Glacy J, Putnam K, Godfrey S, Falzon L, Mauger B, Samson D, et al. Treatments for Seasonal Allergic Rhinitis. AHRQ Comparative Effectiveness Reviews. Rockville (MD)2013.

27. "Draft Guidance for Industry: Allergic Rhinitis: Clinical Development Programs for Drug Products", Food and Drug Administration, CDER. http://wwwfdagov/cder/guidance/indexhtm. 2000. 
28. Allergic Rhinitis: developping drug products for treatment. Guidance for industry.U.S. Department of Health and Human Services Food and Drug Administration Center for Drug Evaluation and Research (CDER) February 2016 Clinical/Medical Revision https://wwwfdagov/downloads/drugs/guidances/ucm 071293 pdf. 2016.

29. Katial RK, Salapatek AM, Patel P. Establishing the onset of action of intranasal corticosteroids: is there an ideal study design? Allergy Asthma Proc. 2009;30(6):595-604.

30. Patel P, D'Andrea C, Sacks HJ. Onset of action of azelastine nasal spray compared with mometasone nasal spray and placebo in subjects with seasonal allergic rhinitis evaluated in an environmental exposure chamber. Am J Rhinol. 2007;21(4):499503.

31. Bousquet J, Meltzer EO, Couroux P, Koltun A, Kopietz F, Munzel U, et al. Onset of Action of the Fixed Combination Intranasal AzelastineFluticasone Propionate in an Allergen Exposure Chamber. J Allergy Clin Immunol Pract. 2018;6(5):1726-32.

32. Patel P, Roland PS, Marple BF, Benninger PJ, Margalias H, Brubaker M, et al. An assessment of the onset and duration of action of olopatadine nasal spray. Otolaryngol Head Neck Surg. 2007;137(6):918-24.

33. Salapatek AM, Lee J, Patel D, D'Angelo P, Liu J, Zimmerer RO, Jr., et al. Solubilized nasal steroid (CDX-947) when combined in the same solution nasal spray with an antihistamine (CDX-313) provides improved, fast-acting symptom relief in patients with allergic rhinitis. Allergy Asthma Proc. 2011;32(3):221-9.

34. Horak F, Zieglmayer UP, Zieglmayer R, Kavina A, Marschall K, Munzel U, et al. Azelastine nasal spray and desloratadine tablets in pollen-induced seasonal allergic rhinitis: a pharmacodynamic study of onset of action and efficacy. Curr Med Res Opin. 2006;22(1):151-7.

35. Zieglmayer P, Zieglmayer R, Bareille P, Rousell V, Salmon E, Horak F. Fluticasone furoate versus placebo in symptoms of grass-pollen allergic rhinitis induced by exposure in the Vienna Challenge Chamber. Curr Med Res Opin. 2008;24(6):1833-40.

36. Murdoch RD, Bareille P, Ignar D, Miller SR, Gupta A, Boardley R, et al. The improved efficacy of a fixed-dose combination of fluticasone furoate and levocabastine relative to the individual components in the treatment of allergic rhinitis. Clin Exp Allergy. 2015;45(8):1346-55.

37. Sleurs K, Seys S, Bousquet J, Fokkens W, Gorris S, Pugin B, et al. Mobile health tools for the management of chronic respiratory diseases. Allergy. 2019.
38. Bousquet J, Hellings PW, Agache I, Bedbrook A, Bachert C, Bergmann KC, et al. ARIA 2016: Care pathways implementing emerging technologies for predictive medicine in rhinitis and asthma across the life cycle. Clin Transl Allergy. 2016;6:47.

39. Menditto E, Guerriero F, Orlando V, Crola C, Di Somma C, Illario M, et al. Self-Assessment of Adherence to Medication: A Case Study in Campania Region Community-Dwelling Population. J Aging Res. 2015;2015:682503.

40. Bonini M. Electronic health (e-Health): emerging role in asthma. Curr Opin Pulm Med. 2017;23(1):21-6.

41. Pizzulli A, Perna S, Florack J, Pizzulli A, Giordani $\mathrm{P}$, Tripodi S, et al. The impact of telemonitoring on adherence to nasal corticosteroid treatment in children with seasonal allergic rhinoconjunctivitis. Clin Exp Allergy. 2014;44(10):1246-54.

42. Price D, Scadding G, Ryan D, Bachert C, Canonica GW, Mullol J, et al. The hidden burden of adult allergic rhinitis: UK healthcare resource utilisation survey. Clin Transl Allergy. 2015;5:39.

43. Bousquet J, Murray R, Price D, Somekh D, Munter L, Phillips J, et al. The allergic allergist behaves like a patient. Ann Allergy Asthma Immunol. 2018;121(6):741-2.

44. Bousquet J, Arnavielhe S, Bedbrook A, AlexisAlexandre G, Eerd Mv, Murray R, et al. Treatment of allergic rhinitis using mobile technology with real world data: The MASK observational pilot study. Allergy. 2018:sous presse.

45. Bedard A, Basagana X, Anto JM, Garcia-Aymerich $\mathrm{J}$, Devillier $\mathrm{P}$, Arnavielhe $\mathrm{S}$, et al. Mobile technology offers novel insights into the control and treatment of allergic rhinitis: The MASK study. J Allergy Clin Immunol. 2019;144(1):135-43 e6.

46. Patel P, Patel D, Kunjibettu S, Hall N, Wingertzahn MA. Onset of action of ciclesonide once daily in the treatment of seasonal allergic rhinitis. Ear Nose Throat J. 2008;87(6):340-53.

47. Wallace DV, Dykewicz MS, Bernstein DI, BlessingMoore J, Cox L, Khan DA, et al. The diagnosis and management of rhinitis: an updated practice parameter. J Allergy Clin Immunol. 2008;122(2 Suppl):S1-84.

48. Roberts G, Xatzipsalti M, Borrego LM, Custovic A, Halken S, Hellings PW, et al. Paediatric rhinitis: position paper of the European Academy of Allergy and Clinical Immunology. Allergy. 2013;68(9):1102-16.

49. Scadding GK, Kariyawasam HH, Scadding G, Mirakian R, Buckley RJ, Dixon T, et al. BSACI guideline for the diagnosis and management of allergic and non-allergic rhinitis (Revised Edition 2017; First edition 2007). Clin Exp Allergy. 2017;47(7):856-89. 
50. Carr W, Bernstein J, Lieberman P, Meltzer E, Bachert C, Price D, et al. A novel intranasal therapy of azelastine with fluticasone for the treatment of allergic rhinitis. J Allergy Clin Immunol. 2012;129(5):1282-9 e10.

51. Meltzer EO. Pharmacotherapeutic strategies for allergic rhinitis: matching treatment to symptoms, disease progression, and associated conditions. Allergy Asthma Proc. 2013;34(4):301-11.

52. Seidman MD, Gurgel RK, Lin SY, Schwartz SR, Baroody FM, Bonner JR, et al. Clinical practice guideline: allergic rhinitis executive summary. Otolaryngol Head Neck Surg. 2015;152(2):197-206.

53. Seidman MD, Gurgel RK, Lin SY, Schwartz SR, Baroody FM, Bonner JR, et al. Clinical practice guideline: Allergic rhinitis. Otolaryngol Head Neck Surg. 2015;152(1 Suppl):S1-43.

54. Bachert C, Bousquet J, Hellings P. Rapid onset of action and reduced nasal hyperreactivity: new targets in allergic rhinitis management. Clin Transl Allergy. 2018;8:25.

55. Church MK, Maurer M, Simons FE, BindslevJensen $\mathrm{C}$, van Cauwenberge $\mathrm{P}$, Bousquet $\mathrm{J}$, et al. Risk of first-generation $\mathrm{H}(1)$-antihistamines: a GA(2)LEN position paper. Allergy. 2010;65(4):45966.

56. Halken S, Larenas-Linnemann D, Roberts G, Calderon MA, Angier E, Pfaar O, et al. EAACI guidelines on allergen immunotherapy: Prevention of allergy. Pediatr Allergy Immunol. 2017;28(8):728-45.

57. Bonertz A, Roberts G, Slater JE, Bridgewater J, Rabin RL, Hoefnagel $M$, et al. Allergen manufacturing and quality aspects for allergen immunotherapy in Europe and the United States: An analysis from the EAACI AIT Guidelines Project. Allergy. 2018;73(4):816-26.

58. Roberts G, Pfaar O, Akdis CA, Ansotegui IJ, Durham SR, Gerth van Wijk R, et al. EAACI Guidelines on Allergen Immunotherapy: Allergic rhinoconjunctivitis. Allergy. 2018;73(4):765-98.

59. Ryan D, Gerth van Wijk R, Angier E, Kristiansen M, Zaman H, Sheikh A, et al. Challenges in the implementation of the EAACI AIT guidelines: A situational analysis of current provision of allergen immunotherapy. Allergy. 2018;73(4):827-36.
60. Pfaar O, Bachert C, Bufe A, Buhl R, Ebner C, Eng $\mathrm{P}$, et al. Guideline on allergen-specific immunotherapy in IgE-mediated allergic diseases: S2k Guideline of the German Society for Allergology and Clinical Immunology (DGAKI), the Society for Pediatric Allergy and Environmental Medicine (GPA), the Medical Association of German Allergologists (AeDA), the Austrian Society for Allergy and Immunology (OGAI), the Swiss Society for Allergy and Immunology (SGAI), the German Society of Dermatology (DDG), the German Society of Oto- Rhino-Laryngology, Head and Neck Surgery (DGHNO-KHC), the German Society of Pediatrics and Adolescent Medicine (DGKJ), the Society for Pediatric Pneumology (GPP), the German Respiratory Society (DGP), the German Association of ENT Surgeons (BV-HNO), the Professional Federation of Paediatricians and Youth Doctors (BVKJ), the Federal Association of Pulmonologists (BDP) and the German Dermatologists Association (BVDD). Allergo J Int. 2014;23(8):282-319.

61. Muraro A, Roberts G, Halken S, Agache I, Angier E, Fernandez-Rivas M, et al. EAACI guidelines on allergen immunotherapy: Executive statement. Allergy. 2018;73(4):739-43.

62. Zielen S, Devillier P, Heinrich J, Richter H, Wahn U. Sublingual immunotherapy provides long-term relief in allergic rhinitis and reduces the risk of asthma: A retrospective, real-world database analysis. Allergy. 2018;73(1):165-77.

63. Devlin N, Parkin D. Does NICE have a costeffectiveness threshold and what other factors influence its decisions? A binary choice analysis. Health Econ. 2004;13(5):437-52.

64. Meadows A, Kaambwa B, Novielli N, Huissoon A, Fry-Smith A, Meads C, et al. A systematic review and economic evaluation of subcutaneous and sublingual allergen immunotherapy in adults and children with seasonal allergic rhinitis. Health Technol Assess. 2013;17(27):vi, xi-xiv, 1-322.

65. Bousquet J, Khaltaev N, Cruz AA, Denburg J, Fokkens WJ, Togias A, et al. Allergic Rhinitis and its Impact on Asthma (ARIA) 2008 update (in collaboration with the World Health Organization, GA(2)LEN and AllerGen). Allergy. 2008;63 Suppl $86: 8-160$.

66. Larenas-Linnemann DES, Antolin-Amerigo D, Parisi C, Nakonechna A, Luna-Pech JA, Wedi B, et al. National clinical practice guidelines for allergen immunotherapy: An international assessment applying AGREE-II. Allergy. 2018;73(3):664-72.

67. Bonertz A, Roberts GC, Hoefnagel M, Timon M, Slater JE, Rabin RL, et al. Challenges in the implementation of EAACI guidelines on allergen immunotherapy: A global perspective on the regulation of allergen products. Allergy. 2018;73(1):64-76. 
68. Committee for medicinal products for human use (CPMP). Guideline on allergen products: production and quality issues. EMEA/CHMP/BWP/304831/2007. London, 20 November 2008. 2008.

69. Kowalski ML, Ansotegui I, Aberer W, Al-Ahmad M, Akdis M, Ballmer-Weber BK, et al. Risk and safety requirements for diagnostic and therapeutic procedures in allergology: World Allergy Organization Statement. World Allergy Organ J. 2016;9(1):33.

70. Bachert C, Larche M, Bonini S, Canonica GW, Kundig $\mathrm{T}$, Larenas-Linnemann $\mathrm{D}$, et al. Allergen immunotherapy on the way to product-based evaluation-a WAO statement. World Allergy Organ J. 2015;8(1):29.

71. Wheatley L, Wood R, Nadeau K, Liu A, Zoratti E, Bacharier L, et al. Mind the gaps: Clinical trial concepts to address unanswered questions in aeroallergen immunotherapy. An NIAID/AHRQ workshop. J Allergy Clin Immunol. 2019;in press.

72. Bousquet J, Lockey R, Malling HJ, Alvarez-Cuesta E, Canonica GW, Chapman MD, et al. Allergen immunotherapy: therapeutic vaccines for allergic diseases. World Health Organization. American academy of Allergy, Asthma and Immunology. Ann Allergy Asthma Immunol. 1998;81(5 Pt 1):401-5.

73. Didier A, Malling HJ, Worm M, Horak F, Jager S, Montagut A, et al. Optimal dose, efficacy, and safety of once-daily sublingual immunotherapy with a 5grass pollen tablet for seasonal allergic rhinitis. J Allergy Clin Immunol. 2007;120(6):1338-45.

74. Nelson H, Blaiss M, Nolte H, Wurtz SO, Andersen JS, Durham SR. Efficacy and safety of the SQstandardized grass allergy immunotherapy tablet in mono- and polysensitized subjects. Allergy. 2013;68(2):252-5.

75. Durham SR, Emminger W, Kapp A, de Monchy JG, Rak S, Scadding GK, et al. SQ-standardized sublingual grass immunotherapy: confirmation of disease modification 2 years after 3 years of treatment in a randomized trial. J Allergy Clin Immunol. 2012;129(3):717-25 e5.

76. Cox L, Larenas-Linnemann D, Lockey RF, Passalacqua G. Speaking the same language: The World Allergy Organization Subcutaneous Immunotherapy Systemic Reaction Grading System. J Allergy Clin Immunol. 2010;125(3):569-74, 74 e174 e7.

77. Passalacqua G, Baena-Cagnani CE, Bousquet J, Canonica GW, Casale TB, Cox L, et al. Grading local side effects of sublingual immunotherapy for respiratory allergy: speaking the same language. J Allergy Clin Immunol. 2013;132(1):93-8.
78. Baiardini I, Puggioni F, Menoni S, Boot JD, Diamant Z, Braido F, et al. Patient knowledge, perceptions, expectations and satisfaction on allergen-specific immunotherapy: a survey. Respir Med. 2013;107(3):361-7.

79. Nam YH, Lee SK. Physician's recommendation and explanation is important in the initiation and maintenance of allergen immunotherapy. Patient Prefer Adherence. 2017;11:381-7.

80. Chivato $\mathrm{T}$, Alvarez-Calderon $\mathrm{P}$, Panizo $\mathrm{C}$, Abengozar R, Alias C, Al-Baech A, et al. Clinical management, expectations, and satisfaction of patients with moderate to severe allergic rhinoconjunctivitis treated with SQ-standardized grass-allergen tablet under routine clinical practice conditions in Spain. Clin Mol Allergy. 2017;15:1.

81. Skoner DP, Blaiss MS, Dykewicz MS, Smith N, Leatherman B, Bielory L, et al. The Allergies, Immunotherapy, and RhinoconjunctivitiS (AIRS) survey: patients' experience with allergen immunotherapy. Allergy Asthma Proc. 2014;35(3):219-26.

82. Pitsios C, Dietis N. Ways to increase adherence to allergen immunotherapy. Curr Med Res Opin. 2018:1-9.

83. Bender BG, Lockey RF. Solving the Problem of Nonadherence to Immunotherapy. Immunol Allergy Clin North Am. 2016;36(1):205-13.

84. Bachert C, Gräfin-von-Strachwitz-Helmstatt K. Zur Diskussion gestellt: Der Arzt und die Spezifische Immuntherapie im Spannungsfeld von Leitlinie, Wirtschaftlichkeit und Medizinrecht. Allergologie. 2016;39:381-8.

85. Kuehl BL, Abdulnour S, O'Dell M, Kyle TK. Understanding the role of the healthcare professional in patient self-management of allergic rhinitis. SAGE Open Med. 2015;3:2050312115595822.

86. Bosnic-Anticevich S, Kritikos V, Carter V, Yan KY, Armour C, Ryan D, et al. Lack of asthma and rhinitis control in general practitioner-managed patients prescribed fixed-dose combination therapy in Australia. J Asthma. 2018;55(6):684-94.

87. Bosnic-Anticevich S, Costa E, Menditto E, Lourenco O, Novellino E, Bialek S, et al. ARIA pharmacy 2018 "Allergic rhinitis care pathways for community pharmacy". Allergy. 2018.

88. Jutel M, Papadopoulos NG, Gronlund H, Hoffman HJ, Bohle B, Hellings P, et al. Recommendations for the allergy management in the primary care. Allergy. 2014;69(6):708-18.

89. Finlay I, Egner W. Allergy--will we ever meet the unmet need? J R Soc Med. 2010;103(11):430-1. 
90. Hellings PW, Fokkens WJ, Bachert C, Akdis CA, Bieber T, Agache I, et al. Positioning the principles of precision medicine in care pathways for allergic rhinitis and chronic rhinosinusitis - A EUFOREAARIA-EPOS-AIRWAYS ICP statement. Allergy. 2017;72(9):1297-305.

91. Pinnock H, Thomas M, Tsiligianni I, Lisspers K, Ostrem A, Stallberg B, et al. The International Primary Care Respiratory Group (IPCRG) Research Needs Statement 2010. Prim Care Respir J. 2010;19 Suppl 1:S1-20.

92. Shehata Y, Ross M, Sheikh A. Undergraduate allergy teaching in a UK medical school: comparison of the described and delivered curriculum. Prim Care Respir J. 2007;16(1):16-21.

93. Alvarez-Cuesta E, Bousquet J, Canonica GW, Durham SR, Malling HJ, Valovirta E. Standards for practical allergen-specific immunotherapy. Allergy. 2006;61 Suppl 82:1-20.

94. Landi M, Meglio P, Praitano E, Lombardi C, Passalacqua G, Canonica GW. The perception of allergen-specific immunotherapy among pediatricians in the primary care setting. Clin Mol Allergy. 2015;13(1):15.

95. Canonica GW, Bachert C, Hellings $\mathrm{P}$, Ryan D, Valovirta E, Wickman $\mathrm{M}$, et al. Allergen Immunotherapy (AIT): a prototype of Precision Medicine. World Allergy Organ J. 2015;8(1):31.

96. Canonica GW, Ansotegui IJ, Pawankar R, SchmidGrendelmeier P, van Hage M, Baena-Cagnani CE, et al. A WAO - ARIA - GA(2)LEN consensus document on molecular-based allergy diagnostics. World Allergy Organ J. 2013;6(1):17.

97. Lee J, Kronborg C, O'Hehir RE, Hew M. Who's at risk of thunderstorm asthma? The ryegrass pollen trifecta and lessons learnt from the Melbourne thunderstorm epidemic. Respir Med. 2017;132:1468.

98. O'Hehir RE, Varese NP, Deckert K, Zubrinich CM, van Zelm MC, Rolland JM, et al. Epidemic Thunderstorm Asthma Protection with Five-Grass Pollen Tablet Sublingual Immunotherapy: A Clinical Trial. Am J Respir Crit Care Med. 2018;198(1):126-8.

99. Pitsios C, Demoly P, Bilo MB, Gerth van Wijk R, Pfaar O, Sturm GJ, et al. Clinical contraindications to allergen immunotherapy: an EAACI position paper. Allergy. 2015;70(8):897-909.

100.Global Strategy for Asthma Management and Prevention (2018 update). wms-GINA-2018-reportV13-002pdf. 2018.
101. Summary of product characteristics. Acarizax 12 SQ-HDM oral lyophilisate. European Medicines Agency. https://mrictsmrpeu/Human/Downloads/DE_H_1947_001_FinalS PCpdf. 2016.

102. Amaral R, Fonseca JA, Jacinto T, Pereira AM, Malinovschi A, Janson C, et al. Having concomitant asthma phenotypes is common and independently relates to poor lung function in NHANES 20072012. Clin Transl Allergy. 2018;8:13.

103. Masuyama K, Okamoto Y, Okamiya K, Azuma R, Fujinami T, Riis B, et al. Efficacy and safety of SQ house dust mite sublingual immunotherapy-tablet in Japanese children. Allergy. 2018.

104.Penagos M, Eifan AO, Durham SR, Scadding GW. Duration of Allergen Immunotherapy for LongTerm Efficacy in Allergic Rhinoconjunctivitis. Curr Treat Options Allergy. 2018;5(3):275-90.

105. Valovirta E, Petersen TH, Piotrowska T, Laursen MK, Andersen JS, Sorensen HF, et al. Results from the 5-year SQ grass sublingual immunotherapy tablet asthma prevention (GAP) trial in children with grass pollen allergy. J Allergy Clin Immunol. 2018;141(2):529-38 e13.

106. Moller C, Dreborg S, Ferdousi HA, Halken S, Host A, Jacobsen L, et al. Pollen immunotherapy reduces the development of asthma in children with seasonal rhinoconjunctivitis (the PAT-study). J Allergy Clin Immunol. 2002;109(2):251-6.

107. Kristiansen M, Dhami S, Netuveli G, Halken S, Muraro A, Roberts G, et al. Allergen immunotherapy for the prevention of allergy: A systematic review and meta-analysis. Pediatr Allergy Immunol. 2017;28(1):18-29.

108. Bozek A, Kolodziejczyk K, Kozlowska R, Canonica GW. Evidence of the efficacy and safety of house dust mite subcutaneous immunotherapy in elderly allergic rhinitis patients: a randomized, double-blind placebo-controlled trial. Clin Transl Allergy. 2017;7:43

109.Bousquet J, Arnavielhe S, Bedbrook A, Fonseca J, Morais Almeida M, Todo Bom A, et al. The Allergic Rhinitis and its Impact on Asthma (ARIA) score of allergic rhinitis using mobile technology correlates with quality of life: The MASK study. Allergy. 2018;73(2):505-10.

110.Pfaar O, Demoly P, Gerth van Wijk R, Bonini S, Bousquet J, Canonica GW, et al. Recommendations for the standardization of clinical outcomes used in allergen immunotherapy trials for allergic rhinoconjunctivitis: an EAACI Position Paper. Allergy. 2014;69(7):854-67. 